\title{
15. HYDROTHERMAL ALTERATION OF THE UPPER-MANTLE SECTION AT HESS DEEP1
}

\author{
Catherine Mével $^{2}$ and Chrysi Stamoudi ${ }^{2}$
}

\begin{abstract}
Short sections of mantle rocks were drilled at Site 895 during Ocean Drilling Program Leg 147 at Hess Deep, where tectonism has exposed lower crust and mantle generated at the equatorial East Pacific Rise. These mantle sections consist of harzburgites and dunites that are impregnated by mafic liquids and crosscut by gabbroic and diabase dikes. The mantle rocks are thought to represent the Moho transition zone. This suite of samples has been extensively altered by interaction with seawater. Ultramafics are largely serpentinized. Gabbroic rocks are strongly hydrated and recrystallized and partly rodingitized. Most recrystallization occurred under static conditions. Secondary assemblages in the gabbroic rocks provide evidence for at least three successive stages of recrystallization. High-temperature amphiboles are scarce and likely represent an early episode that also crystallized secondary clinopyroxene in veins. The major episode of alteration occurred under greenschist facies conditions and is characterized by coronas of serpentine, tremolite, chlorite, and prehnite. The coronas are contemporaneous with the bulk of serpentinization. A later alteration episode produced thomsonite and potassic micas after plagioclase, and late carbonate veins. Comparison of temperatures determined from mineral assemblages with thermal models of the oceanic lithosphere suggests that peak alteration took place at some distance from the ridge axis. Massive penetration of seawater in the mantle is therefore interpreted to result from the rifting of the crust generated at the East Pacific Rise by the Cocos-Nazca propagator.
\end{abstract}

\section{INTRODUCTION}

All mantle rocks exposed on the seafloor are generally serpentinized, between $50 \%$ and $100 \%$ as a result of interaction with seawater (Bonatti, 1976; Prichard, 1979; Bonatti et al., 1984; Kimball et al., 1985; Agrinier et al., 1988; Tartarotti et al., 1995). Evaluating the extent of serpentinization in the oceanic mantle is important because it has strong physical and chemical implications. Serpentinization is accompanied by a decrease in density (possibly down to $2.5 \mathrm{~g} / \mathrm{cm}^{3}$ ) which modifies the physical properties of the mantle, its gravity signature, and seismic velocities. Therefore, serpentinization strongly influences geophysical measurements. Moreover, serpentinization is accompanied by chemical fluxes that affect chemical exchange between seawater and the oceanic lithosphere. Finally, some authors suggested that oceanic tectonics is due, in part, to serpentine protrusions and diapiric uplifting of low density serpentines (Bonatti, 1976; Francis, 1981; Francheteau et al., 1990). However, direct access to the mantle underlying the crust is impossible with the present drilling technology, and indirect measurements of crustal properties are difficult to interpret. Therefore, to evaluate the extent of serpentinization at depth, it is important to understand the mechanisms which allow seawater penetration into the mantle.

To answer this question, one important parameter is the temperature at which serpentinization occurs. It may shed light on the distance to the ridge axis where the mantle was located when it was massively hydrated. Previous studies have already shown that serpentinization is generally not restricted to the seafloor, when mantle rocks have been exposed by tectonics, but rather occurs at significant depths and elevated temperature (e.g., Bonatti et al., 1984; Kimball et al., 1985).

The tectonic regime is also an important parameter because faulting plays a major role in seawater penetration. The extent of serpen-

'Mével, C., Gillis, K.M., Allan, J.F., and Meyer, P.S. (Eds.), 1996. Proc. ODP, Sci. Results, 147: College Station, TX (Ocean Drilling Program).

${ }^{2}$ Laboratoire de Pétrologie, CNRS URA 736, UPMC, 4 place Jussieu, 75252 Paris cedex 05, France. Mével: cam@ccr.jussieu.fr tinization at depth is certainly a factor of spreading rate that influences the regime of faulting and the thickness of the crust. Serpentinized mantle outcrops have been recognized as a normal part of the building of oceanic lithosphere in slow-spreading ridges (Cannat, 1993). However, little is known about serpentinization in fast-spreading ridges, such as the East Pacific Rise, where mantle rocks crop out less frequently and in more anomalous zones.

This paper deals with a suite of mantle rocks drilled at Site 895 in Hess Deep during Ocean Drilling Program (ODP) Leg 147. The rocks consist of serpentinized peridotites that have been impregnated and intruded by mafic melts. Therefore, interaction with seawater produced serpentinites after ultramafic rocks as well as metagabbros. The secondary mineralogy in the metagabbros is more sensitive to temperature than serpentine. To decipher the temperature conditions of serpentinization, this study is mainly oriented toward the metagabbros.

\section{GEOLOGICAL SETTING AND SAMPLING}

Hess Deep is located at the tip of the Cocos-Nazca Ridge, which propagates into the crust generated at the East Pacific Rise (EPR), at a half spreading rate of $13 \mathrm{~mm} / \mathrm{yr}$ (Searle and Francheteau, 1986; Lonsdale, 1988) (Fig. 1). At this latitude, the EPR opens at a half rate of $65 \mathrm{~mm} / \mathrm{yr}$. The structure of the deep is characterized by an intrarift ridge that is oriented east-west and culminates at approximately 3000 $m$ depth, perpendicular to the EPR and parallel to the Cocos-Nazca Ridge. This intrarift ridge is located north of the deep that reaches $5400 \mathrm{~m}$ depth. The slopes of this ridge were explored with the French submersible Nautile during the Nazcopac cruise in 1988 (Francheteau et al., 1990). On the southern flank, gabbroic rocks crop out on the western side, whereas mantle peridotites make up the lower slopes on the eastern side (Hekinian et al., 1993). Site 895 was placed directly on the peridotite outcrops to obtain sections of the mantle generated at the EPR. Six holes were drilled at this site (Gillis, Mével, Allan, et al., 1993), among which only three produced sections over $30 \mathrm{~m}$ (Holes $895 \mathrm{C}, 895 \mathrm{D}$, and $895 \mathrm{E}$ ). Figure 2 summarizes the lithologies recovered at this site. 


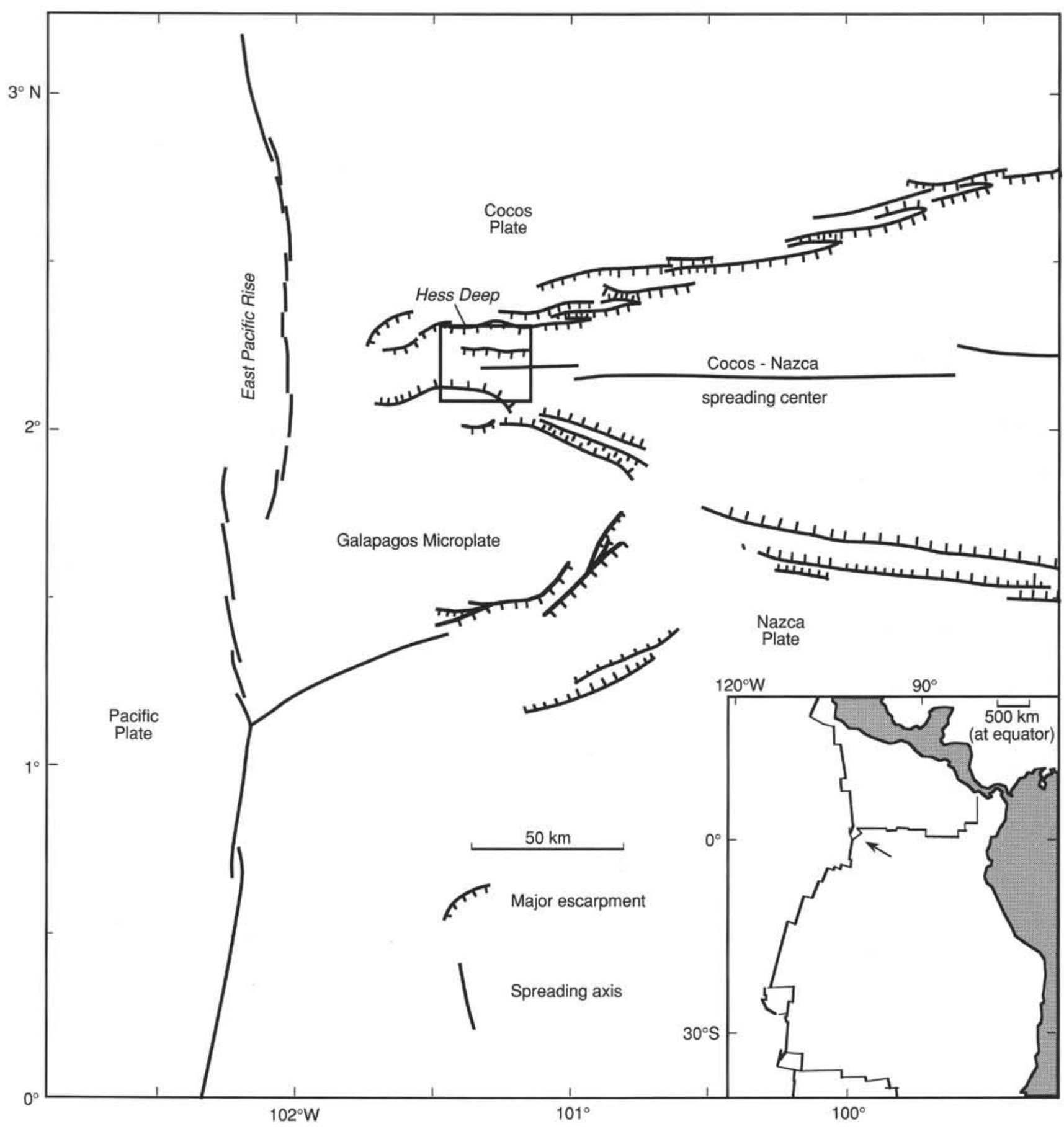

Figure 1. Location map of Hess Deep (Gillis, Mével, Allan, et al., 1993).

The rock types recovered in the different holes are similar but vary in proportion (Fig. 2). They consist of harzburgites, dunites, troctolites, olivine gabbros, gabbros, and gabbronorites, as well as a few basaltic dikes. The few contacts that were observed suggest that harzburgite grades into dunite by the disappearance of orthopyroxene. Dunites are in turn impregnated by mafic liquids that crystallized plagioclase plus minor clinopyroxene. In a single sample, the percentage of such crystallization products varies from a few percent to $50 \%$ (see fig. 6 in Gillis, Mével, Allan, et al., 1993). The impregnated rocks may be called troctolites, although they might be partially residual in origin (Boudier et al., this volume; Dick and Natland, this volume; Arai and Matsukage, this volume). But some troctolites as well as the gabbros display crosscutting relationships with the ultramafics suggesting a magmatic origin, with possibly a few olivine xenocrysts. The gabbroic rocks are very heterogeneous, with grain size varying from a few hundred of microns to several centimeters in a single piece (Fig. 3A). This intermixing between harzburgites, dunites, and mafic rocks is interpreted to be the Moho transition zone (Boudier et al., this volume), as observed in ophiolite complexes (Boudier and Nicolas, in press). Therefore, it is likely that the mantle sections recovered were originally located at a depth in the order of $5-6 \mathrm{~km}$ beneath the seafloor. 


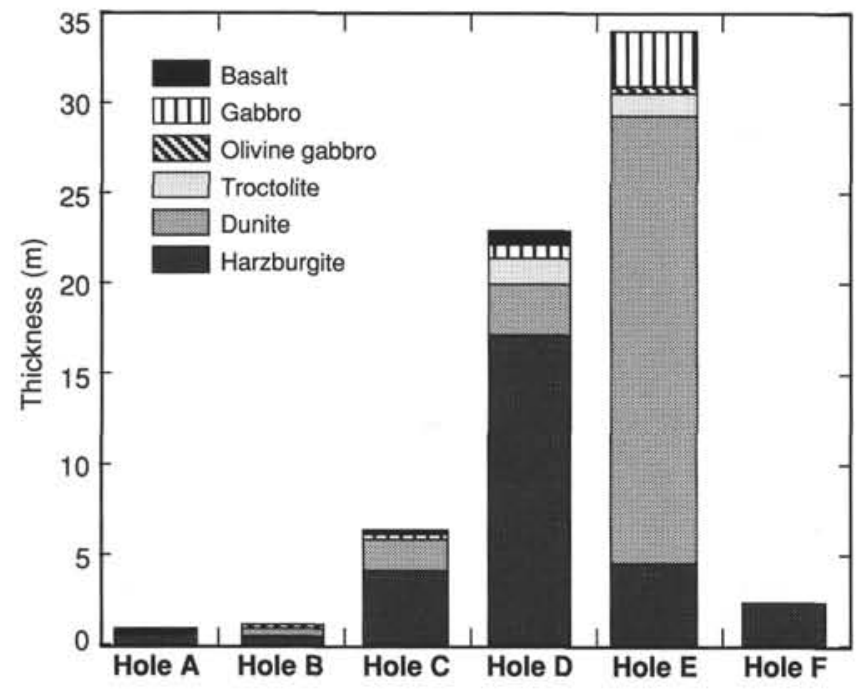

Figure 2. Summary of the lithology of holes drilled at Site 895 (Gillis, Mével, Allan, et al., 1993).

The mechanism responsible for the uplifting of the intrarift ridge is not well understood. Francheteau et al. (1990) propose two alternative models: (1) vertical uplifting due to serpentine diapirism; (2) uplifting related to low-angle detachment faulting during rifting at the tip of the Cocos-Nazca propagator.

\section{PETROGRAPHY OF ALTERED ROCKS}

Ultramafic and gabbroic rocks from the mantle section display pervasive recrystallization. Initial descriptions on board ship emphasized that there is no systematic variation in alteration intensity with depth. By contrast, there is a tendency for rocks from Hole 895E to be more altered that rocks from Hole 895E (Gillis, Mével, Allan, et al., 1993). This difference in alteration intensity is related to lithology: pyroxenes tend to be more resistant to alteration than olivines. Therefore, among ultramafic rocks, dunites are systematically more altered than harzburgites. Because dunites are more abundant in Hole $895 \mathrm{E}$ than in $895 \mathrm{D}$, rocks from Hole $895 \mathrm{E}$ tend to be more altered.

Thirty-eight samples, mostly from Holes 895D and 895E, were selected for detailed studies of secondary assemblages (Table 1). Textural relationships show that most recrystallization occurred under static conditions. In ultramafic rocks, the only evidence of deformation is thin sheared surfaces coated with serpentine. A few gabbroic rocks, however, display oriented fabrics in hand specimen that seem to result from early deformation. The secondary phases present in the rocks are generally not oriented, showing that they grew after deformation ended. In a few samples, a weak foliation is defined by the growth of chlorite and amphibole that surround small porphyroclasts.

Most of the samples, particularly the ultramafics, are crosscut by vein networks. However, structural measurements on board ship showed that, as opposed to Site 894 gabbros, the veins have no systematic orientation. The veins tend to be more diffuse and not as organized. The different types of veins will be described together with the rocks they crosscut. Table 1 gives a summary of the secondary phases observed in the samples studied.

\section{Ultramafic Rocks}

\section{Harzburgites}

Harzburgites contain olivine, orthopyroxene, and minor spinel and clinopyroxene. They often display a porphyroclastic texture, with large elongated orthopyroxene and streaks of spinel. In some samples, small xenomorphic clinopyroxene crystals are interstitial between olivine crystals or surround and penetrate large orthopyroxene porphyroclasts. This texture is suggestive of impregnation and is similar to textures described by Girardeau and Francheteau (1993).

Recrystallizations in harzburgites are rather simple, and typical of oceanic (or ophiolitic) peridotites (e.g., Prichard, 1979; Komor et al., 1985; Hébert et al., 1990). Secondary mineralogy is dominated by serpentine. Olivine is partly or completely replaced by serpentine and magnetite intergrowths forming the classical mesh texture (Prichard, 1979). Typically, magnetite occurs at the edge of serpentine cores that replace olivine kernels. However, magnetite is sometimes aligned in a network that consists of subparallel streaks. This distribution may suggest a preferential direction for fluid circulation during serpentinization. The proportion of serpentine with respect to magnetite seems to vary, but may simply reflect the grain size of secondary magnetite which varies from the order of 10 microns to less than a micron. The mineralogical assemblage replacing orthopyroxene depends on the intensity of alteration. In the less altered samples, talc is abundant and occurs as rims or in fractures crosscutting the orthopyroxene crystals. When alteration is more extensive, large bastite crystals pseudomorph orthopyroxene. Amphibole is scarce and may occur as colorless needles around the orthopyroxene pseudomorphs (Fig. 3B). Clinopyroxene remains essentially fresh or is partially replaced by serpentine and acicular colorless amphibole. Spinel seems to be generally fresh, retaining its brownish color.

\section{Dunites}

Dunites are composed mostly of olivine, with very minor spinel and occasional orthopyroxene. Dunites grade into troctolites by the appearance of interstitial plagioclase and minor clinopyroxene. Olivine in dunites is preserved in rare kernels that display the typical mesh texture of serpentine plus magnetite. Again, magnetite is distributed along a preferential direction. In many samples, euhedral olivine pseudomorphs are isolated in a matrix of fibrous, colorless serpentine that seems to be contemporaneous with the alteration of the olivine crystals (Fig. 3C). Where present, orthopyroxene is replaced by bastite. Brown spinel is either fresh, or altered to a black secondary magnetite-rich spinel.

The presence of interstitial plagioclase drastically changes the nature of secondary phases. The plagioclase may be partly preserved, but most of the time it is completely recrystallized. The resulting rocks are characterized by coronitic reactions between olivine and plagioclase, involving serpentine, amphibole, chlorite, and calc-silicates. The coronas are better developed in troctolites and olivine gabbros and will be described in more detail later. Where present, clinopyroxene either remains unaltered or is involved in the coronitic reactions.

\section{Veins in ultramafic rocks}

Both harzburgites and dunites are crosscut by numerous veins, showing complex relationships that are not always easy to decipher. As a whole, these veins do not represent more than a few percent of the rocks' volume. A first generation of generally large veins has sinuous contacts with the enclosing rock and may be connected to a dense network of oblique thinner veins. These large veins are filled with serpentine, often fibrous, and magnetite. They seem to be contemporaneous of the bulk of the serpentinization, and the network of thinner veins may result from an adjustment to volume increase. The first generation is crosscut by later veins that may have sharp contacts with earlier serpentine, and are filled with a more birefringent variety of serpentine that grew perpendicular to the vein edges. Later veins are filled with serpentine plus brucite plus possibly clay minerals and aragonite. 

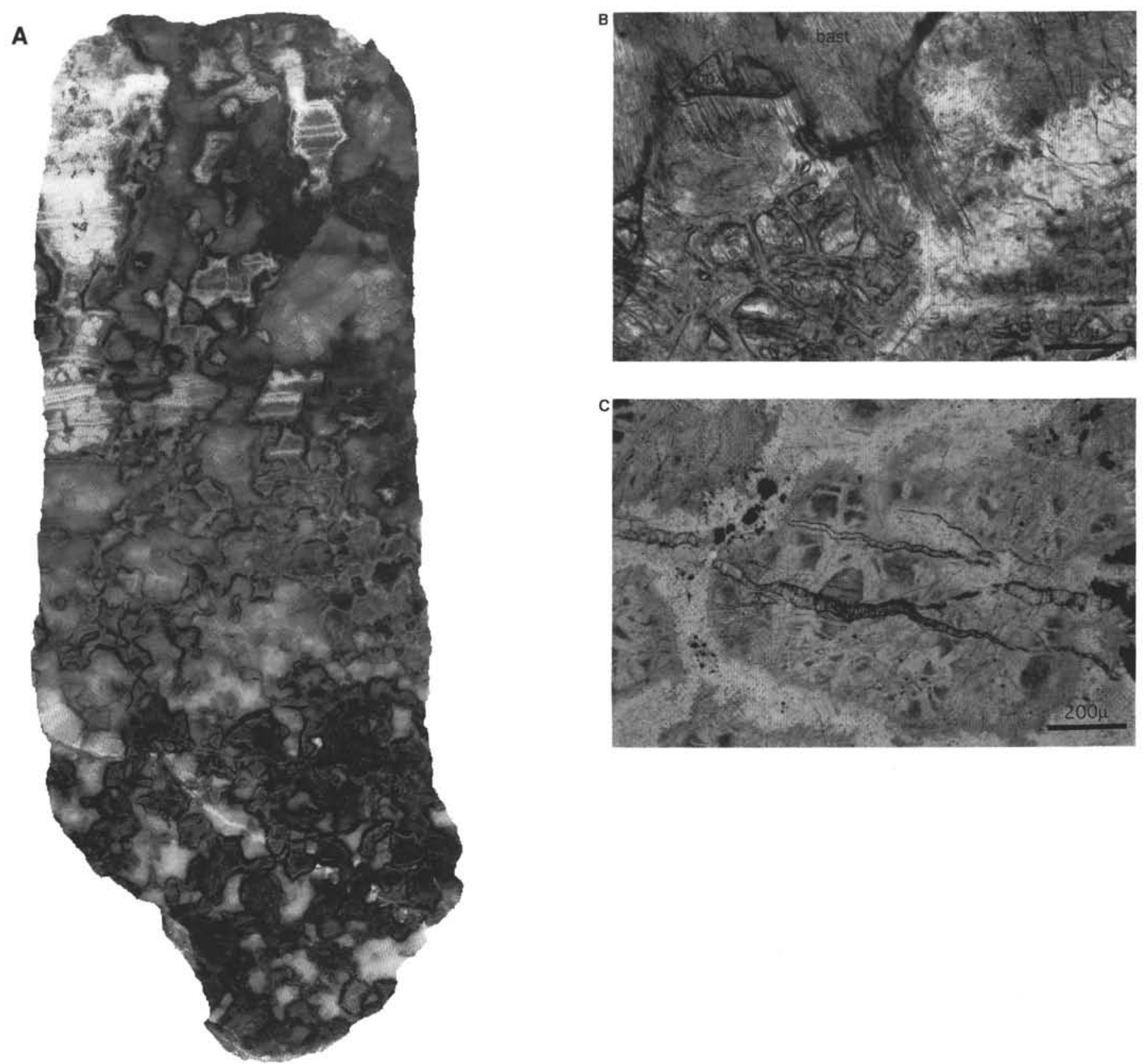

Figure 3. Photographs of selected samples. A. Gabbroic sample displaying a heterogeneous texture with areas rich in olivine (corona texture) and clinopyroxene. Sample 147-895E-2R-2, 0-16 cm. B. Harzburgite, photomicrograph. A large orthopyroxene, completely replaced by bastite (bast) is rimmed by clinopyroxene (cpx) at the contact with olivine (olv). The cpx is largely preserved, and rimmed by tremolite needles (tr). Sample 147-895D-3R-1, 64-69 cm, parallel nicols. C. Dunite, photomicrograph. Alteration texture showing olivine pseudomorphs, with the typical serpentine mesh texture, isolated in a serpentine + magnetite matrix. Late veins filled with a more birefringent variety of serpentine crosscut the rock. Sample 147-895E-6R-3, 80-84 cm, parallel nicols.

\section{Gabbroic Rocks}

The limit between ultramafic and gabbroic rocks is often difficult to draw because in a single core, the amount of plagioclase may vary from a few percent to almost $50 \%$. Some of the mafic rocks may be at least partially residual in origin, made up of mantle olivine impregnated with magmatic liquids, while others are likely completely magmatic (Dick and Natland, this volume; Boudier, this volume). However, they are described together because the presence of plagioclase, even in a small amount, has a strong influence on secondary mineralogy. The transition from troctolites to olivine gabbros is also progressive, by increasing the amount of clinopyroxene.
Typically, the gabbroic rocks recovered within the mantle section at Site 895 are much more altered than those from Hole $894 \mathrm{G}$ which represent the upper section of the lower crust. At Site 895, alteration varies between $40 \%$ and $100 \%$ and is characterized by an abundance of hydrous minerals such as chlorite, amphibole, and prehnite. Most of the alteration occurred under static conditions, although a few samples may have been deformed during an early stage. Only in a few samples (Table 1) is a weak foliation defined by chlorite and/or acicular amphibole.

The troctolites consist of euhedral olivine and rare spinel enclosed in plagioclase and accessory clinopyroxene. Generally, clinopyroxene occurs as thin rims around olivine crystals, but may form small 

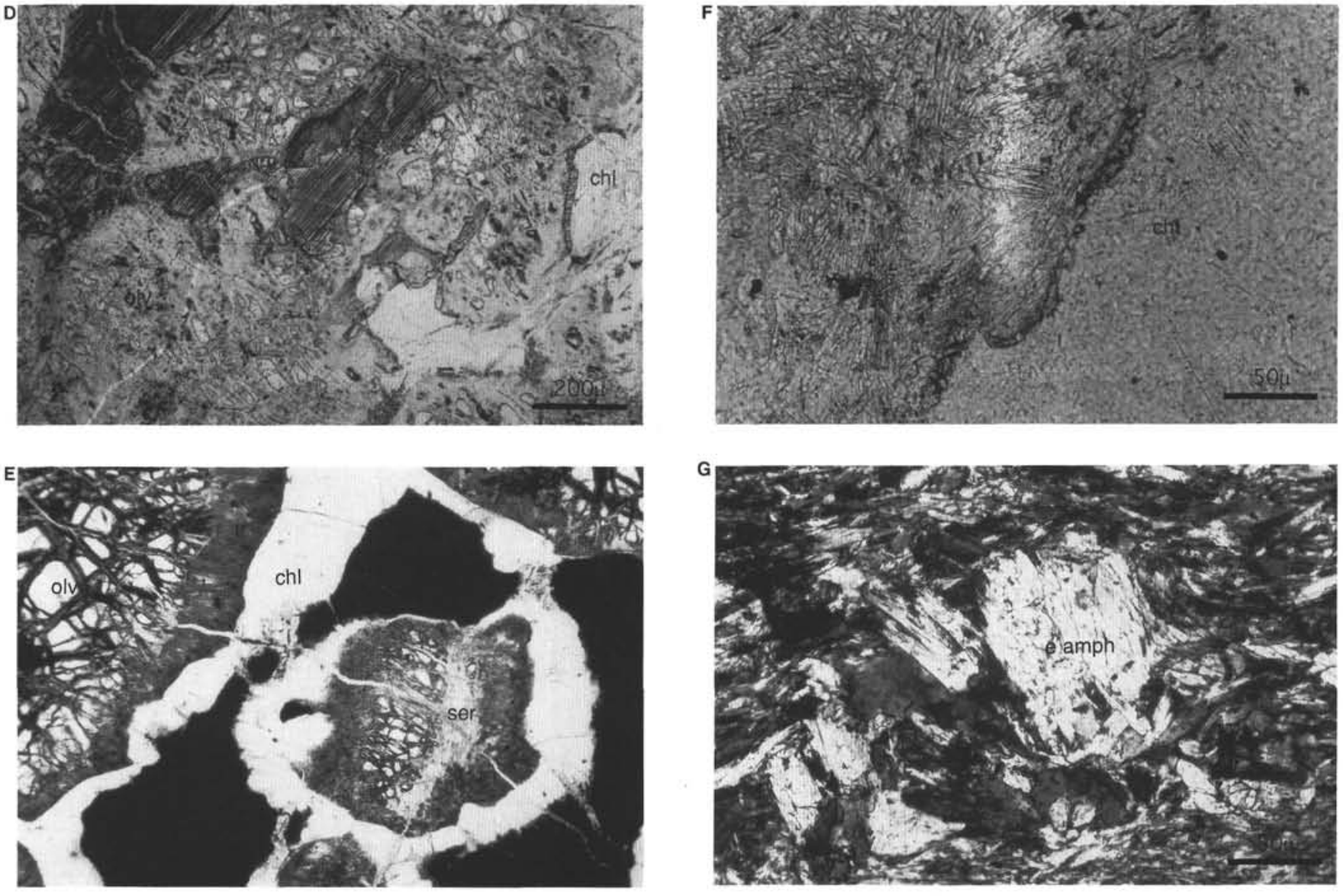

Figure 3 (continued). D. Olivine gabbro, photomicrograph. Partly altered olivine is isolated by interstitial plagioclase and clinopyroxene. Clinopyroxene (cpx) may form large crystals or narrow rims around the olivine (olv). The plagioclase is completely replaced by chlorite (chl). Sample 147-895C-4R-1, 8-11 cm, parallel nicols. E. Troctolite. Photomicrograph of a typical coronitic texture. Olivine (olv), partly replaced by serpentine + magnetite (ser), is rimmed by acicular tremolite (tr), which radiates from the contact with the altered plagioclase. The plagioclase is completely replaced by chlorite (chl) at the contact with the olivine, and a dark microcrystalline aggregate consisting dominantly of prehnite (pr) in the center. Sample 147-895D-7R-1, 56-60 cm, parallel nicols. F. Troctolite. Photomicrograph of the contact between an olivine pseudomorph and a plagioclase pseudomorph. The small rim of clinopyroxene that marks the contact is still visible, although almost completely altered. The olivine is replaced by tremolite (tr), the plagioclase by chlorite (chl). Sample 147-895E-6R-1, 85-87 cm, parallel nicols. G. Photomicrograph of a deformed gabbro. Small porphyroclast of early amphibole (e amph) preserved in a foliation defined by tremolite needles. Sample 147-895E-1R-1, 58-63 cm, crossed nicols.

individual interstitial crystals (Fig. 3D). In the olivine gabbros, the clinopyroxene forms large interstitial crystals. There is no significant difference in the alteration style of both rock types.

There is very little evidence of an early metamorphic episode predating the major corona-producing event (see infra). In one deformed sample (Sample 147-895E-1R-1, 140-144 cm), small porphyroclasts of colorless amphibole are preserved in a weak foliation defined by tremolite needles (Fig. 3G). In a few samples, narrow clinopyroxene veins crosscut olivine and/or plagioclase crystals (Fig. $3 \mathrm{H}$ ). These veins seem to dissolve in the chlorite replacing the plagioclase, suggesting that chloritization postdates their formation. Moreover, they are often partly altered to an acicular colorless amphibole. Secondary clinopyroxene also occurs in the gabbros from Site 894. However, it pseudomorphs the primary pyroxene and is related to a pervasive episode of alteration (Gillis, Mével, Allan, et al., 1993; Manning et al., 1993). In this case, it is obviously related to a fracturing episode. Another feature of some gabbroic rocks are small veins filled with deepgreen amphibole that cross cut plagioclase. These veins have no sharp contacts with the plagioclase but rather correspond to alignments of randomly oriented needles (Fig. 3I). In Sample 147-895E$2 \mathrm{R}-2,10-15 \mathrm{~cm}$, they seem to replace clinopyroxene in veins, but they evidently predate the formation of chlorite.
In most of the rocks, the main episode of recrystallization produced coronas from the reaction between plagioclase and olivine. Typically, olivine is replaced by a mesh of serpentine and magnetite, surrounded by a rim of acicular tremolite, which marks the contact with the plagioclase (Fig. 3E). Large magmatic clinopyroxene crystals are well preserved, with only narrow rims of secondary amphibole. When clinopyroxene forms only thin rims, it may be either preserved or recrystallized acicular amphibole (Fig. 3F). The plagioclase is replaced by chlorite that sometimes surrounds a microcrystalline brownish core. Microprobe studies of the brownish cores show that it consists essentially of prehnite, mixed with epidote (clinozoisite) and possibly hydrogrossular.

Veins of serpentine, chlorite and/or prehnite occur in the gabbroic rocks. The chlorite veins seem mostly contemporaneous of the corona formation because they are connected with chlorite replacing plagioclase. The prehnite veins, by contrast, cut through the whole samples and seem to postdate the corona formation. In several samples (Table 1), plagioclase is partly replaced by a late zeolite or by "sericite," a flaky microcrystalline mineral (Fig. 3J), in relation with late fracturing. The prehnite veins might be contemporaneous with the formation of these minerals. Finally, late veins crosscut the whole samples and are filled with carbonates and possibly clay minerals. 

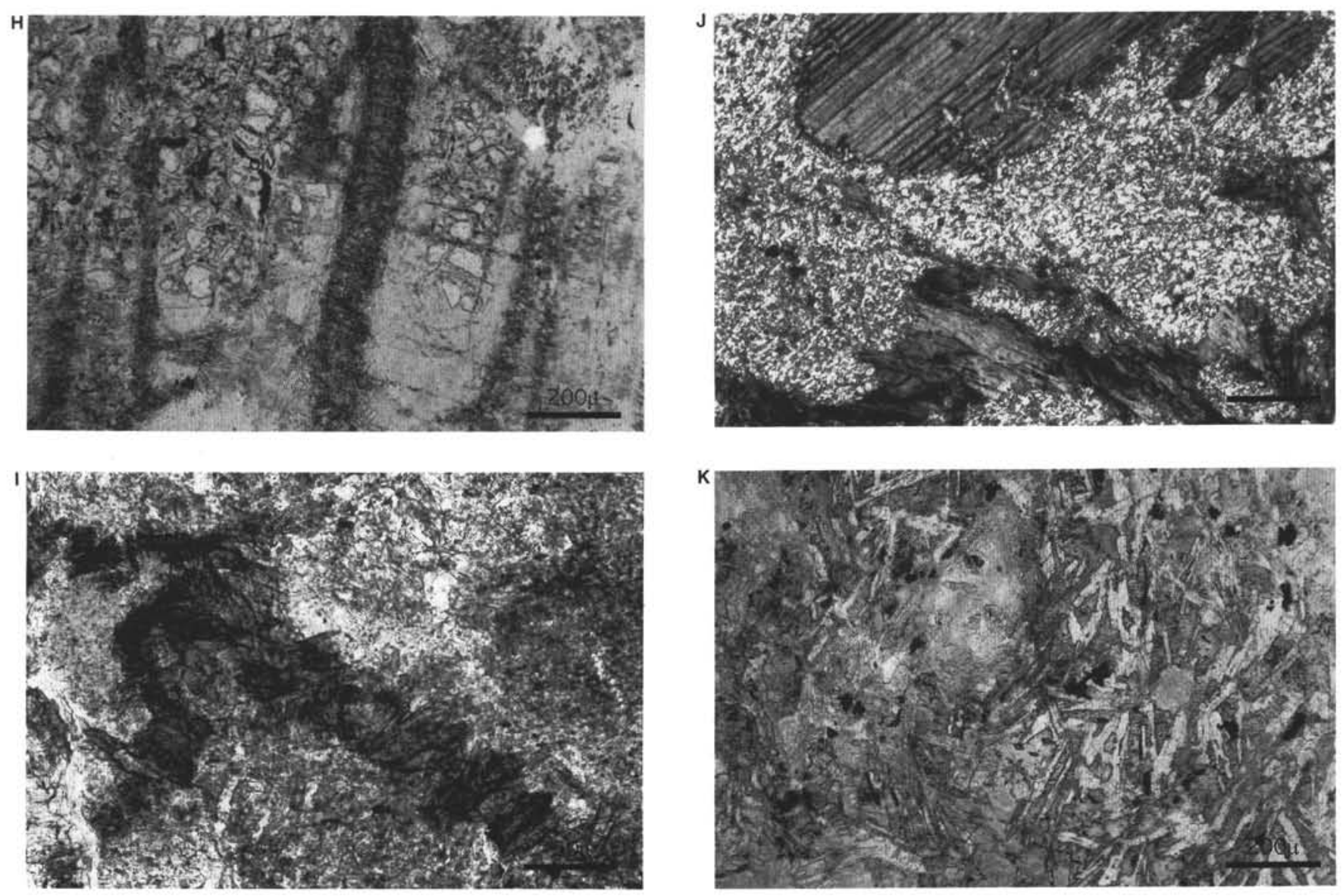

Figure 3 (continued). H. Troctolite, photomicrograph. Parallel veins of clinopyroxene crosscutting partially serpentinized olivine. The clinopyroxene is partly altered to acicular amphibole. Sample 147-895E-6R-1, 85-87 cm, parallel nicols. I. Gabbro. Photomicrograph showing sinuous veins filled with needles of green amphibole (ga) crosscutting an altered crystal of plagioclase. Sample 147-895E-2R-1, 128-133 cm, parallel nicols. J. Troctolite. Photomicrograph of a plagioclase partly replaced by flaky, microcrystalline, sericite-like crystals. Sample $147-895 \mathrm{E}-1 \mathrm{R}-1,58-63 \mathrm{~cm}$, crossed nicols. K. Diabasic dike, photomicrograph. The diabasic texture is still identifiable. Laths of plagioclase are surrounded by brown amphibole, and amphibole aggregates that may replace clinopyroxene. Sample 147-895C-4R-3, 56-59 cm, parallel nicols.

In conclusion, the petrography of secondary assemblages suggests the following evolution with time:

1. Crystallization of the amphibole which now forms the porphyroclasts; veins filled with secondary clinopyroxene; green amphibole?

2. Corona formation which produced serpentine, tremolite, chlorite, prehnite, and epidote, possibly hydrogrossular

3. Replacement of plagioclase by zeolite and/or sericite; prehnite veins?

4. Late veins of calcite + clay minerals

The occurrence of abundant calc-silicate minerals such as prehnite and zeolites raises the issue whether the gabbroic rocks are rodingites.

\section{Diabase Dike}

A few diabase dikes were sampled. Their metamorphic character is very different from the rocks previously described. The rock texture is still magmatic (Fig. 3K), with euhedral crystals of plagioclase enclosed in large areas of interstitial amphibole. An early amphibole is characteristically brown, and forms beautiful, limpid crystals. Its texture suggests that it might be late magmatic. Later amphibole is greenish, and may replace clinopyroxene. Oxides are partly altered to sphene. As opposed to the enclosing gabbros, the diabases lack abundant chlorite.

\section{MINERAL CHEMISTRY}

Mineral chemical analyses were performed with a Cameca microprobe at CAMPARIS, Université Pierre et Marie Curie, Paris. A combination of natural silicates and oxides was used as standards. Counting time varied between 10 and $20 \mathrm{~s}$, depending on the element concentrations, and the beam current was $10 \mathrm{vA}$. Depending on the minerals analyzed, different beam sizes were used. The beam was focused to $2-3 \mu \mathrm{m}$ for the resistant minerals (olivine, plagioclase, amphibole, pyroxenes, chlorite). It was unfocused to $10 \mu \mathrm{m}$ for the minerals easily destroyed (serpentine, zeolite, prehnite). In the case of microcrystalline aggregates, the analyses correspond to an aggregate of crystals.

\section{Serpentines}

As already pointed out by many studies (Agrinier et al., 1988; Hébert et al., 1990), the composition of serpentine is strongly dependent on the texture and the mineral it replaces (Table 2). 
Table 1. Summary of the petrography of the samples studied.

\begin{tabular}{|c|c|c|c|c|}
\hline $\begin{array}{l}\text { Core, section, } \\
\text { interval }(\mathrm{cm})\end{array}$ & Rock type & Primary minerals & Secondary minerals & Observations \\
\hline $\begin{array}{l}\text { 147-895A- } \\
\text { IR-1,58-61 }\end{array}$ & harzburgite & olv, opx, cpx, sp, cpx i & ser, mt, trem & \\
\hline $\begin{array}{l}147-895 \mathrm{C}- \\
1 \mathrm{R}-1,0-7 \\
3 \mathrm{R}-1,94-97 \\
4 \mathrm{R}-1,8-11 \\
4 \mathrm{R}-3,56-59\end{array}$ & $\begin{array}{l}\text { harzburgite } \\
\text { harzburgite } \\
\text { impr dunite } \\
\text { Diabase }\end{array}$ & $\begin{array}{l}\text { olv, (opx), sp, cpx i } \\
\text { olv, (opx), cpx, sp } \\
\text { olv, cpx, sp, (pl) } \\
\text { pl, b amph, ox, zr }\end{array}$ & $\begin{array}{l}\text { ser, mt, trem } \\
\text { ser, mt, late veins of aragonite } \\
\text { ser, mt, trem, chl, pr? } \\
\text { hb, act, zeo }\end{array}$ & $\begin{array}{l}\text { thick serpentine vein } \\
\text { thick serpentine vein } \\
\text { late zeolite veins }\end{array}$ \\
\hline $\begin{array}{l}147-895 \mathrm{D} \\
2 \mathrm{R}-2,81-84 \\
3 \mathrm{R}-1,64-69 \\
4 \mathrm{R}-3,90-94 \\
7 \mathrm{R}-1,56-60 \\
7 \mathrm{R}-2,42-46 \\
8 \mathrm{R}-1,100-103 \\
8 \mathrm{R}-2,126-130 \\
10 \mathrm{~W}-1,36-43 \\
10 \mathrm{~W}-1,54-61\end{array}$ & $\begin{array}{l}\text { harzburgite } \\
\text { harzburgite } \\
\text { harzburgite } \\
\text { pl-dunite } \\
\text { harzburgite } \\
\text { ols gabbro } \\
\text { dunite } \\
\text { troctolite } \\
\text { olv gabbro }\end{array}$ & $\begin{array}{l}\text { olv, opx, cpx, sp } \\
\text { olv, opx, cpx, sp } \\
\text { olv, opx, cpx, tc } \\
\text { olv, cpx, sp, (pl) } \\
\text { olv, opx, cpx, sp } \\
\text { olv, cpx, pl } \\
\text { (olv), sp } \\
\text { (olv), cpx, sp, pl } \\
\text { olv, cpy, sp, pl }\end{array}$ & $\begin{array}{l}\text { ser, mt, trem } \\
\text { ser, mt, tc, trem } \\
\text { ser, mt, trem, chl, pr } \\
\text { ser, mt, tc } \\
\text { ser, mt, trem, cpx2, chl, pr, thom } \\
\text { ser, mt } \\
\text { ser?, mt, trem, chl, } \\
\text { hb s.l., ser, mt, trem, chl, pr, thom }\end{array}$ & $\begin{array}{l}\text { abundant veins of chlorite } \\
\text { late brucite veins } \\
\text { clinozoite, hydrogrossular? }\end{array}$ \\
\hline $\begin{array}{l}\text { I47-895E- } \\
\text { IR-1, 26-28 } \\
\text { IR-1, 29-36 } \\
\text { IR-1, 58-63 } \\
\text { IR-1, 140-144 } \\
\text { IR-3, 101-104 } \\
\text { IR-3, 112-115 } \\
\text { 2R-1, 128-133 } \\
\text { 2R-2, 10-15 } \\
\text { 3R-1, 67-70 } \\
\text { 4R-1, 20-22 } \\
\text { 5R-2, 104-107 } \\
\text { 6R-1, 85-87 } \\
\text { 6R-1, 123-125 } \\
\text { 6R-3, 80-84 } \\
\text { 6R-5, 56-61 } \\
\text { 6R-5, 111-115 } \\
\text { 7R-3, 33-37 } \\
\text { 7R-4, 11-14 } \\
\text { 8R-3, 88-93 }\end{array}$ & $\begin{array}{l}\text { troctolite } \\
\text { troctolite } \\
\text { troctolite } \\
\text { troctolite? } \\
\text { olv gabbro } \\
\text { troctolite? } \\
\text { troctolite? } \\
\text { troctolite } \\
\text { dunite } \\
\text { olv gabbro? } \\
\text { dunite } \\
\text { troctolite } \\
\text { dunite } \\
\text { dunite } \\
\text { harzburgite } \\
\text { dunite } \\
\text { harzburgite } \\
\text { harzburgite } \\
\text { dunite }\end{array}$ & $\begin{array}{l}\text { (olv), (pl) } \\
\text { (olv), cpx, pl } \\
\text { (olv), cpx, sp, pl } \\
\text { (olv?), cpx, pl } \\
\text { (olv), (sp), pl } \\
\text { (olv?), cpx, sp, pl } \\
\text { olv, cpx, pl } \\
\text { olv, cpx, pl } \\
\text { olv, sp } \\
\text { (olv), (cpx?), (opx?), pl } \\
\text { (olv), sp } \\
\text { olv, cpx, sp, pl } \\
\text { olv, sp } \\
\text { olv, sp } \\
\text { olv, opx, sp } \\
\text { olv, sp } \\
\text { olv, opx, sp } \\
\text { olv, opx, cpx, sp } \\
\text { (olv), (sp) }\end{array}$ & $\begin{array}{l}\text { ser, mt, trem, chl, pr, thom } \\
\text { trem, g amph, chl } \\
\text { ser, mt, trem, g amph, chl, pr } \\
\text { trem, g amph, pr, mica } \\
\text { trem, chl, ser } \\
\text { trem, chl } \\
\text { trem, g amph, cpx2, chl, pr, thom } \\
\text { ser, mt, tc, trem, g amph, cpx2, pr, thom, mica } \\
\text { ser, mt } \\
\text { ser, mt, tc, trem, chl } \\
\text { ser, mt } \\
\text { ser, mt, hb, trem, cpx2, chl, pr } \\
\text { ser, mt } \\
\text { ser, mt } \\
\text { ser, mt } \\
\text { ser, mt } \\
\text { ser, mt } \\
\text { ser, mt, tc } \\
\text { ser, mt }\end{array}$ & $\begin{array}{l}\text { abundant mica flakes replacing pl } \\
\text { foliation underlined by chl, trem } \\
\text { foliation underlined by trem, chl } \\
\text { abundant calc-silicates } \\
\text { foliation underlined by trem, chl } \\
\text { white matrix between olv grains } \\
\text { network of cpx veins } \\
\text { late orange brucite vein } \\
\text { thick serpentine veins } \\
\text { brucite in veins } \\
\text { sheared serpentine veins } \\
\text { vein network oblique to HT foliation }\end{array}$ \\
\hline $\begin{array}{l}147-895 \mathrm{~F}- \\
2 \mathrm{R}-1,55-59 \\
2 \mathrm{R}-1,137-142 \\
2 \mathrm{R}-2,43-48\end{array}$ & $\begin{array}{l}\text { dunite } \\
\text { dunite } \\
\text { dunite }\end{array}$ & $\begin{array}{l}\text { (olv), (sp) } \\
\text { olv, sp } \\
\text { olv, sp }\end{array}$ & $\begin{array}{l}\text { ser, } \mathrm{mt} \\
\text { ser, } \mathrm{mt} \\
\text { ser, } \mathrm{mt}\end{array}$ & $\begin{array}{l}\text { late orange brucite veins? } \\
\text { brucite? }\end{array}$ \\
\hline
\end{tabular}

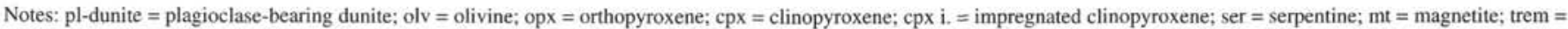
tremolite; $\mathrm{tc}=$ talc; $\mathrm{cpx} 2=$ secondary clinopyroxene; $\mathrm{hb}=$ hornblende; $\mathrm{act}=$ actinolite; $\mathrm{sp}=$ spinel; $\mathrm{chl}=\mathrm{chlorite} ; \mathrm{pr}=\mathrm{prehnite} ; \mathrm{g}$ amph = green amphibole; $\mathrm{b}$ amph = brown amphibole; zeo = zeolite. Primary minerals between parentheses are no longer present. ? = identification questionable.

In ultramafic rocks, typically, serpentine after olivine contains no aluminum and no chromium but an average of $0.30 \mathrm{wt} \% \mathrm{NiO}$, which is about the nickel content of fresh olivine. By contrast, bastite contains some aluminum, and is rich in chromium and devoid of nickel. The $\mathrm{XMg}\left(\mathrm{XMg}=\mathrm{Mg} /\left(\mathrm{Fe}^{2+}+\mathrm{Mg}\right)\right.$ of serpentine is variable, from 0.90 to 0.96 , and heterogeneous numbers can be found in a single olivine pseudomorph. The variation of the $\mathrm{XMg}$ may be related to the presence of submicroscopic grains of magnetite, which are analyzed together with the serpentine. Serpentine in the first generation of veins is systematically enriched in aluminum. Aluminum content varies from $2 \mathrm{wt} \%$ up to $8 \mathrm{wt} \%$ in the matrix between olivine pseudomorphs in dunites (Table 2, analyses 10,11). In the gabbroic rocks, serpentine occurs after olivine or in veins. As opposed to the ultramafics, serpentine after olivine may contain some aluminum. The $\mathrm{XMg}$ of serpentine tends to be lower, and reaches down to 0.75 . Chlorine content in serpentine may reach up to $0.30 \mathrm{wt} \%$. But, as opposed to the serpentinites from the Galicia Bank (Agrinier et al., 1988), no systematic distribution of chlorine could be identified.

\section{Plagioclase}

Plagioclase seems mostly primary, although, as a rule, it is very turbid. Its composition varies between anorthite (An) An93 and An85. No secondary more sodic compositions were found. However, in a few samples, secondary more calcic compositions seem to occur. For instance, in Sample 147-895E-2R-2, 10-15 cm, primary plagioclase has a composition of An85 while secondary plagioclase, An90, is associated to the secondary clinopyroxene.

\section{Clinopyroxene}

Secondary clinopyroxene has been found mainly in veins in gabbroic rocks and exceptionally as rims around primary clinopyroxene. A few crystals of apparently primary clinopyroxene (on the basis of textural relationships) were analyzed for comparison with secondary clinopyroxene (Table 3 ) and plotted in a Wo:En:Fs ternary diagram (Fig. 4A). Primary clinopyroxene (Fig. 4B) contains an average of 3 wt $\% \mathrm{FeO}$, and also contains appreciable amount of titanium (0.05$0.25 \mathrm{Ti})$ and aluminum $(0.08-0.18 \mathrm{Al})$. By contrast, secondary clinopyroxene in veins typically contains very little aluminum and titanium (Fig. 4D). Similar relationships between magmatic and secondary pyroxene have been documented in oceanic gabbros (Mével, 1987; Gillis et al., 1993; Vanko and Stakes, 1991). They likely reflect a temperature decrease between crystallization of primary and secondary pyroxene. Another character of the vein clinopyroxene is a strong iron enrichment in two of the samples (Samples 147-895E-2R$1,128-133 \mathrm{~cm}$, and $147-895 \mathrm{E}-2 \mathrm{R}-2,10-15 \mathrm{~cm}$ ); the iron content reaches $10 \mathrm{wt} \% \mathrm{FeO}$ (Fig. 4C). This very high iron value contrasts with the general $\mathrm{Mg}$-rich composition of the mafic minerals.

\section{Amphiboles}

Amphiboles are scarce in ultramafics, but widespread in gabbroic rocks. It is the major secondary mineral of the dike sample that has been studied in the present work. The amphibole analyzed in the harzburgite (Sample 147-895D-3R-1, 64-69 cm) is an almost pure tremolite (Table 4 , analysis 1 ). In the gabbroic rocks, compositions 
Table 2. Representative analyses of serpentines.

\begin{tabular}{|c|c|c|c|c|c|c|c|c|c|c|c|c|c|c|c|c|}
\hline & 1 & 2 & 3 & 4 & 5 & 6 & 7 & 8 & 9 & 10 & 11 & 12 & 13 & 14 & 15 & 16 \\
\hline Hole: & $895 \mathrm{C}$ & $895 \mathrm{C}$ & 895D & 895D & 895D & 895D & 895D & 895D & 895D & 895D & $895 \mathrm{E}$ & $895 \mathrm{E}$ & $895 \mathrm{E}$ & $895 \mathrm{E}$ & 895D & 895D \\
\hline Core, section: & $3 R-1$ & $3 R-1$ & $3 R-1$ & $3 R-1$ & $7 R-2$ & $7 \mathrm{R}-2$ & $8 \mathrm{R}-2$ & $8 \mathrm{R}-2$ & $8 \mathrm{R}-2$ & $8 \mathrm{R}-2$ & $7 \mathrm{R}-4$ & $7 \mathrm{R}-4$ & $6 \mathrm{R}-1$ & $6 \mathrm{R}-1$ & $8 \mathrm{R}-2$ & $7 \mathrm{R}-1$ \\
\hline Interval (cm): & 94-97 & 94-97 & $64-69$ & 64-69 & $42-46$ & $42-46$ & $126-130$ & $126-130$ & $126-130$ & $126-130$ & $11-14$ & $11-14$ & $85-87$ & $85-87$ & $35-38$ & $56-60$ \\
\hline Site: & olv & vein 1 & opx & olv & opx & vein & olv & matrix & matrix & vein & opx & olv & olv & vein & olv & olv \\
\hline $\mathrm{SiO}_{2}$ & 38.66 & 40.17 & 38.18 & 40.40 & 40.97 & 40.45 & 39.50 & 37.37 & 40.14 & 41.49 & 41.17 & 36.67 & 39.33 & 40.81 & 41.80 & 39.35 \\
\hline $\mathrm{TiO}_{2}^{2}$ & 0 & 0 & 0.04 & 0 & 0 & 0.04 & 0 & 0.06 & 0 & 0 & 0.05 & 0 & 0.06 & 0.01 & 0.02 & 0.07 \\
\hline $\mathrm{Al}_{2} \mathrm{O}_{3}$ & 0.04 & 2.77 & 3.25 & 0.27 & 2.73 & 1.06 & 0 & 8.41 & 2.83 & 0 & 2.86 & 0 & 0.13 & 0.71 & 0.98 & 3.63 \\
\hline $\mathrm{Cr}_{2} \mathrm{O}_{3}$ & 0.02 & 0.94 & 1.35 & 0 & 1.42 & 0.05 & 0.05 & 0 & 0.04 & 0.02 & 1.02 & 0 & 0.11 & 0.02 & 0.07 & 0.01 \\
\hline $\mathrm{FeO}$ & 4.96 & 5.30 & 4.97 & 6.34 & 4.49 & 6.31 & 4.48 & 2.72 & 3.02 & 3.84 & 5.49 & 6.85 & 8.33 & 5.77 & 16.15 & 8.85 \\
\hline $\mathrm{MnO}$ & 0.05 & 0.13 & 0.02 & 0.16 & 0.08 & 0.27 & 0.11 & 0.04 & 0.01 & 0 & 0 & 0.13 & 0.15 & 0.12 & 0.06 & 0.08 \\
\hline $\mathrm{MgO}$ & 37.45 & 35.64 & 35.69 & 36.98 & 34.75 & 36.74 & 38.53 & 36.96 & 38.02 & 37.29 & 36.73 & 40.82 & 34.83 & 36.28 & 27.54 & 32.86 \\
\hline $\mathrm{NiO}$ & 0.38 & 0.24 & 0 & 0.10 & 0.09 & 0.05 & 0.37 & 0.12 & 0.04 & 0 & 0.05 & 0.26 & 0.37 & 0.04 & 0.11 & 0.28 \\
\hline $\mathrm{CaO}$ & 0.05 & 0.07 & 0.05 & 0.12 & 1.12 & 0.05 & 0.04 & 0 & 0.03 & 0.10 & 0.06 & 0.03 & 0.09 & 0.04 & 0.12 & 0.24 \\
\hline $\mathrm{Na}_{2} \mathrm{O}$ & 0.03 & 0.01 & 0.01 & 0 & 0.03 & 0.02 & 0.01 & 0.01 & 0.02 & 0.07 & 0.01 & 0 & 0.04 & 0.02 & 0.10 & 0.01 \\
\hline $\mathrm{K}_{2} \mathrm{O}$ & 0.04 & 0.04 & 0.01 & 0 & 0.02 & 0.01 & $\stackrel{0}{0}$ & 0 & 0 & 0.21 & 0 & 0 & 0.01 & 0 & 0.07 & 0 \\
\hline Total & 81.83 & 85.35 & 83.74 & 84.56 & 85.82 & 85.23 & 83.21 & 85.75 & 84.22 & 83.06 & 87.53 & 85.03 & 83.53 & 83.92 & 87.17 & 85.54 \\
\hline $\mathrm{Cl}$ & 0.118 & 0.009 & 0.14 & 0.15 & 0.084 & 0.135 & 0.086 & 0.022 & 0.037 & 0.011 & 0.07 & 0.249 & 0.027 & 0.05 & 0.10 & 0.123 \\
\hline $\mathrm{Si}$ & 1.963 & 1.947 & 1.892 & 1.990 & 1.972 & 1.975 & 1.965 & 1,776 & 1.942 & 2.043 & 1.945 & 1.829 & 1.986 & 2.011 & 2.083 & 1.936 \\
\hline Ti & 0 & & 0.001 & 0 & 0 & 0.001 & 0 & 0.471 & 0.16 & 0 & 0.001 & & 0.0 & & 0.001 & 0.002 \\
\hline Al & 0.002 & 0.158 & 0.189 & 0.015 & 0.154 & 0.060 & 0 & 0.002 & 0 & 0 & 0.158 & 0 & 0.007 & 0.041 & 0.057 & 0.21 \\
\hline $\mathrm{Cr}$ & 0 & 0.036 & 0.053 & 0 & 0.054 & 0.002 & 0 & 0 & & 0 & 0.038 & 0 & 0.004 & & 0.002 & 0 \\
\hline $\mathrm{Fe}$ & 0.210 & 0.214 & 0.206 & 0.261 & 0.180 & 0.258 & 0.186 & 0.108 & 0.122 & 0.158 & 0.217 & 0.285 & 0.352 & 0.238 & 0.673 & 0.364 \\
\hline Mn & 0.002 & 0.005 & 0.001 & 0.007 & 0.003 & 0.011 & 0.004 & 0.001 & 0 & 0 & 0 & 0.005 & 0.006 & 0.005 & 0.002 & 0.003 \\
\hline $\mathrm{Mg}$ & 2.834 & 2.576 & 2.636 & 2.715 & 2.493 & 2.674 & 2.857 & 2.619 & 2.742 & 2.737 & 2.586 & 3.036 & 2.621 & 2.664 & 2.045 & 2.411 \\
\hline $\mathrm{Ni}$ & 0.015 & 0.009 & 0 & 0.004 & 0.003 & 0.001 & 0.015 & 0.004 & 0.001 & 0 & 0.001 & 0.010 & 0.015 & 0.001 & 0.004 & 0.011 \\
\hline $\mathrm{Ca}$ & 0.002 & 0.003 & 0.002 & 0.006 & 0.058 & 0.003 & 0.002 & & 0.001 & 0.005 & 0.003 & 0.001 & 0.005 & 0.002 & 0.006 & 0.012 \\
\hline $\mathrm{Na}$ & 0.003 & 0.001 & 0.001 & 0 & 0.003 & 0.002 & 0.001 & 0.001 & 0.002 & 0.007 & 0.001 & 0 & 0.004 & 0.001 & 0.009 & 0.001 \\
\hline K & 0.002 & 0.002 & & 0 & 0.001 & 0 & 0 & 0 & & 0.013 & & 0 & 0.001 & & 0.004 & 0 \\
\hline Total & 5.038 & 4.956 & 4.985 & 5.001 & 4.925 & 4.992 & 5.034 & 4.986 & 4.977 & 4.966 & 4.954 & 5.170 & 5.007 & 4.968 & 4.892 & 4.955 \\
\hline $\mathrm{Cl}$ & 0.010 & & 0.011 & 0.012 & 0.006 & 0.011 & 0.007 & 0.001 & 0.003 & 0 & 0.005 & 0.021 & 0.002 & 0.004 & 0.008 & 0.010 \\
\hline $\mathrm{XMg}$ & 0.93 & 0.92 & 0.93 & 0.91 & 0.93 & 0.91 & 0.93 & 0.96 & 0.96 & 0.94 & 0.92 & 0.91 & 0.87 & 0.91 & 0.75 & 0.86 \\
\hline
\end{tabular}

Notes: Structural formulae calculated on the basis of 22 oxygens per formula unit. Analyses 1-12: ultramafic rocks; $13-16=$ gabbroic rocks. Olv = serpentine after olivine; op $x=$ serpentine after orthopyroxene. 
Table 3. Representative analyses of clinopyroxenes.

\begin{tabular}{|c|c|c|c|c|c|c|c|}
\hline & 1 & 2 & 3 & 4 & 5 & 6 & 7 \\
\hline Hole: & 895D & 895D & 895D & $895 \mathrm{E}$ & $895 \mathrm{E}$ & $895 \mathrm{E}$ & $895 \mathrm{E}$ \\
\hline Core, section: & $8 R-1$ & $10 \mathrm{~W}-1$ & $10 \mathrm{~W}-1$ & $6 \mathrm{R}-1$ & $2 \mathrm{R}-1$ & $2 \mathrm{R}-2$ & $6 \mathrm{R}-1$ \\
\hline Interval $(\mathrm{cm})$ : & $100-103$ & $54-61$ & $54-61$ & $85-87$ & $128-133$ & $10-15$ & $85-87$ \\
\hline Site: & 1 large & 1 aureole & 1 aureole & 1 aureole & 2 & 2 & 2 \\
\hline $\mathrm{SiO}_{2}$ & 51.53 & 52.06 & 52.91 & 51.17 & 52.00 & 52.97 & 54.90 \\
\hline $\mathrm{TiO}_{2}$ & 0.35 & 0.80 & 0.84 & 0.18 & 0.09 & 0.00 & 0.01 \\
\hline $\mathrm{Al}_{2} \mathrm{O}_{3}$ & 3.11 & 3.28 & 2.83 & 4.29 & 0.42 & 0.26 & 0.08 \\
\hline $\mathrm{Cr}_{2} \mathrm{O}_{3}$ & 0.83 & 0.76 & 0.62 & 1.13 & 0.00 & 0.04 & 0.00 \\
\hline $\mathrm{Fe}_{2} \mathrm{O}_{3}$ & 0.37 & 0 & 0 & 0.20 & 0.00 & 0.00 & 0.00 \\
\hline $\mathrm{FeO}$ & 3.33 & 3.54 & 2.98 & 2.25 & 10.85 & 10.62 & 2.43 \\
\hline $\mathrm{MnO}$ & 0.06 & 0.14 & 0.16 & 0.06 & 0.38 & 0.37 & 0.14 \\
\hline $\mathrm{MgO}$ & 16.25 & 16.82 & 16.42 & 15.52 & 11.08 & 11.41 & 17.17 \\
\hline $\mathrm{CaO}$ & 21.9 & 21.48 & 22.95 & 23.81 & 24.03 & 24.2 & 24.76 \\
\hline $\mathrm{Na}_{2} \mathrm{O}$ & 0.27 & 0.25 & 0.28 & 0.18 & 0.06 & 0.07 & 0.01 \\
\hline $\mathrm{K}_{2} \mathrm{O}$ & 0.06 & 0.01 & 0 & 0.00 & 0.03 & 0.00 & 0.02 \\
\hline Total & 98.1 & 99.18 & 100.03 & 98.84 & 98.98 & 99.98 & 99.53 \\
\hline $\mathrm{Si}$ & 1.916 & 1.910 & 1.925 & 1.888 & 1.991 & 2.002 & 2.005 \\
\hline $\mathrm{Al}^{\mathrm{IV}}$ & 0.083 & 0.089 & 0.074 & 0.111 & 0.008 & 0 & 0 \\
\hline $\mathrm{Al}^{\mathrm{vI}}$ & 0.052 & 0.052 & 0.047 & 0.075 & 0.010 & 0.011 & 0.002 \\
\hline $\mathrm{Ti}$ & 0.009 & 0.022 & 0.023 & 0.005 & 0.001 & 0 & 0 \\
\hline $\mathrm{Cr}$ & 0.024 & 0.022 & 0.018 & 0.033 & 0 & 0.001 & 0 \\
\hline $\mathrm{Fe}^{3+}$ & 0.010 & 0 & 0 & 0.005 & 0 & 0 & 0 \\
\hline $\mathrm{Fe}^{2+}$ & 0.103 & 0.108 & 0.090 & 0.069 & 0.347 & 0.335 & 0.074 \\
\hline $\mathrm{Mn}$ & 0.002 & 0.004 & 0.005 & 0.002 & 0.012 & 0.012 & 0.004 \\
\hline $\mathrm{Mg}$ & 0.901 & 0.92 & 0.891 & 0.853 & 0.632 & 0.643 & 0.934 \\
\hline $\mathrm{Ca}$ & 0.872 & 0.844 & 0.895 & 0.941 & 0.986 & 0.980 & 0.968 \\
\hline $\mathrm{Na}$ & 0.020 & 0.017 & 0.019 & 0.013 & 0.004 & 0.005 & 0 \\
\hline K & 0.003 & 0 & 0 & 0 & 0.001 & 0 & 0.001 \\
\hline Total & 4.000 & 3.994 & 3.991 & 4.000 & 3.999 & 3.993 & 3.993 \\
\hline
\end{tabular}

Notes: Structural formulae calculated on the basis of 6 oxygens per formula unit. Fe ${ }^{3+}$ is estimated by normalizing the cation sum to 4.1 large $=$ large magmatic crystal; 1 aureole $=$ magmatic crystal rimming olivine; 2 = secondary clinopyroxene in veins.

vary largely, depending on the microstructural site. A few Al-rich compositions have been found in some samples. The most characteristic occurs in Sample 147-895E-1R-1, 58-63 cm, in which amphibole forms small porphyroclasts preserved in a foliation defined by tremolite (Fig. 3G). These amphiboles vary from pargasitic hornblende to edenitic hornblende to hornblende s.s. (Fig. 5A). A few other Al-rich compositions occur as small preserved zones in more tremolitic amphiboles. These early amphiboles are highly magnesian and almost completely devoid of titanium, as opposed to the early amphiboles from Hole 894G.

The most widespread amphibole, associated with the coronas, has a tremolite composition, with a XMg about $91-93$ (Fig. 5B). There is no significant difference between the amphibole composition in the coronas and those that define the foliation in Sample 147-895E-1R$1,58-63 \mathrm{~cm}$. This composition is similar to the composition found in the harzburgite.

The green amphibole that forms sinuous veins in plagioclase has a very peculiar composition. It is very depleted in silica ( $\mathrm{Si}=5.9$ to 6.5 per formula unit), and relatively rich in iron $(8 \%-12 \% \mathrm{FeO})$. It falls essentially in the ferroan pargasite and ferroan pargasitic hornblende fields (Fig. 5C). Figure 6 shows the contrast in composition between the magnesian, $\mathrm{Si}$-rich tremolites and the $\mathrm{Fe}$-rich, $\mathrm{Si}$-poor green pargasite-edenite occurring in the same samples. This Fe-rich composition should be compared to the pyroxenes that occur in veins in at least two of the same samples (147-895E-2R-1, 128-133 cm, and $147-895 \mathrm{E}-2 \mathrm{R}-2,10-15 \mathrm{~cm}$ ). This supports the interpretation that at least in some cases, the green, Fe-rich amphiboles recrystallized from earlier secondary clinopyroxene enriched in iron.

Amphibole compositions are very distinct in the basaltic dike. The amphiboles are calcic and follow the pargasite-actinolite trend (Fig. 4D). Their titanium content decreases as their color varies from brown to green. They contain appreciable iron, reflecting the bulk rock composition.

Appreciable amount of chlorine may be present (up to $0.20 \mathrm{wt} \%$ ) in amphibole, especially in early amphibole. But no strong enrichment was observed.

\section{Chlorite}

Chlorite is a widespread secondary phase in gabbros. It is essentially clinochlore and penninite, with a few talc chlorite and ripidolite compositions (Table 5). These various compositions correspond to variations in the silica and iron contents. The compositional variations may occur at the scale of a single sample (e.g., Sample 147$895 \mathrm{E}-2 \mathrm{R}-1,128-133 \mathrm{~cm}$ ). Although not completely systematic, they seem to reflect the site of crystallization: chlorite close to olivine contact tends to be Si- and Mg-rich, whereas chlorite in the center of plagioclase pseudomorphs tends to be Al-rich and Fe-rich (Table 5; Fig. 7).

\section{Talc}

Talc replaces orthopyroxene in harzburgites. It also occurs in two examples of gabbroic rocks that are heavily altered. It is not clear in this case whether talc replaces orthopyroxene or olivine. It contains appreciable amount of aluminum (2-4 wt\%) and iron (generally around $4 \mathrm{wt} \%$ ).

\section{Other Minerals}

Other minerals include prehnite, thomsonite, a flaky mica-like material and late vein-filling. The prehnite is Fe-poor $\left(\mathrm{Fe}_{2} \mathrm{O}_{3}\right.$ tot $<1$ wt\%; Table 6), as already described in oceanic gabbros (Gillis et al., 1993; Bideau et al., 1991). In Sample 147-895D-10W-1, 54-61 cm, clinozoisite has been analyzed (Table 6 , analysis 4 ). The analysis is not very good because the mineral is microcrystalline; however, it is characteristically rich in aluminum compared to the prehnites. It is also devoid of iron. Analyses of thomsonites are shown in Table 6. Thomsonite in oceanic gabbros also has been observed in Hole 735B (Stakes et al., 1991). One peculiar characteristic of a few gabbroic rocks is the presence of a white mica. Because it occurs at very small flakes (Fig. 3J), this white mica proved difficult to analyze. However, 
analysis 10 in Table 6 shows that it contains up to $9 \%$ potassium and that its composition is almost consistent with a muscovite.

\section{DISCUSSION}

The importance of this suite of rocks is the close association of mafic rocks with the ultramafics. While ultramafics give secondary parageneses mainly consisting of serpentine plus magnetite, which is stable under a large temperature range, mafic rocks produce secondary assemblages that contain minerals that more tightly indicate recrystallization temperatures. The close relationships between the mafic and ultramafic rocks suggest that metamorphism affected both lithologies at the same time. Therefore, information from the mafic rocks may be applied to the ultramafic rocks as well.

\section{Temperature Conditions}

In the gabbros, the mineral assemblages suggest than an early episode produced aluminous amphibole (pargasitic hornblende and hornblende) whose composition is characteristic of the amphibolite facies (above $500^{\circ} \mathrm{C}$ ) (Liou et al. 1974; Spear, 1981). Amphiboles of similar composition occur as early background alteration in gabbros from Hole $894 \mathrm{G}$. No textural relationships were observed between this early high-temperature amphibole and the clinopyroxene veins. The composition of secondary clinopyroxene, plotted in a Wo:En:Fs ternary diagram (Fig. 4A), suggests a temperature in the range of $500^{\circ} \mathrm{C}$ (Fig. 4C) (Lindsley and Andersen, 1983; Lindsley, 1983). They are similar to secondary pyroxenes observed in the MARK area (Gillis et al., 1993) as well as in the gabbros from 735B in the Indian Ocean (Stakes et al., 1991). One of their characteristics is iron enrichment, contrasting with the very magnesium-rich character of the rocks. Fluids enriched in iron are necessary to produce these compositions. Similarly, the presence of ferroan pargasite postdating the clinopyroxene veins is difficult to explain. Their peculiar compositions (very depleted in silica and rich in iron) may reflect a local chemical environment.

The typomorphic assemblage, however, is coronas between plagioclase and olivine, and calc-silicates in the plagioclase pseudomorphs. As opposed to coronitic reactions described by Gillis et al.(1993) in the MARK area, these coronas only involve greenschist facies minerals: serpentine, tremolite, chlorite, and calc-silicates (prehnite, epidote, and possibly some hydrogrossular). The stability range of prehnite is approximately between 220 and $430^{\circ}$ (Liou et al, 1983). The composition of amphibole and the presence of chlorite together with prehnite and epidote suggest that the formation temperature was likely in the range of $300-400^{\circ} \mathrm{C}$. Textural relationships suggest that the bulk of serpentinization is contemporaneous with corona formation, which is the major metamorphic episode in the gabbros and corresponds to a massive introduction of water. Typically, serpentinites contain more than $10 \mathrm{wt} \%$ water (Gillis, Mével, Allan, et al., 1993). Stable isotope measurements in the serpentines suggest that serpentinization occurred at relatively high temperature and that the fluid phase is largely seawater (Früh-Green, Plas, and Lécuyer, this volume). Moreover, Agrinier et al (in press) calculated a temperature of serpentinization of $350^{\circ}$ from the equilibrium partitioning of ${ }^{18} \mathrm{O}$ in a serpentine-magnetite pair. This temperature is consistent with the greenschist assemblage of the gabbros.

The following evolution is suggested for these rocks:

- There probably has been an early, high temperature metamorphic episode that produced the Al-rich amphibole, and may additionally have produced secondary clinopyroxene in the fracture network, and possibly green amphibole in veins crosscutting plagioclase. Compared to the high-temperature alteration in the gabbros from the lower crust at Hole 894G, however, the early episode at Site 895 is very restricted in extent. It may be related to deep fluid penetration at

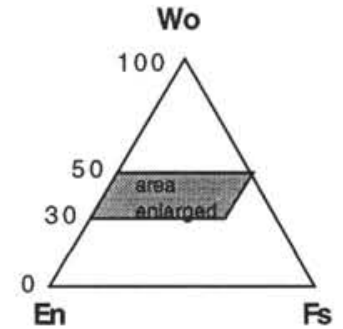

Wo

B

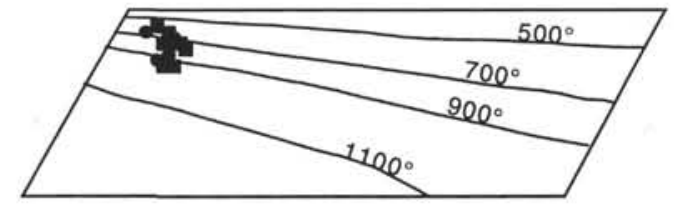

En

FS

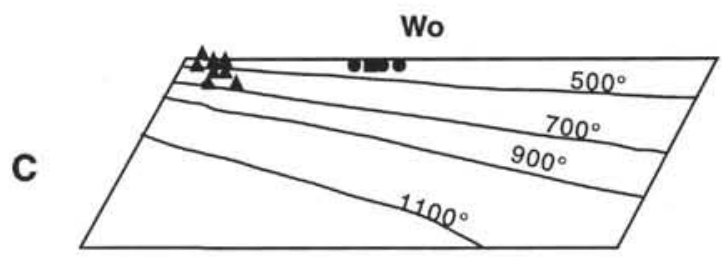

En

Fs

D

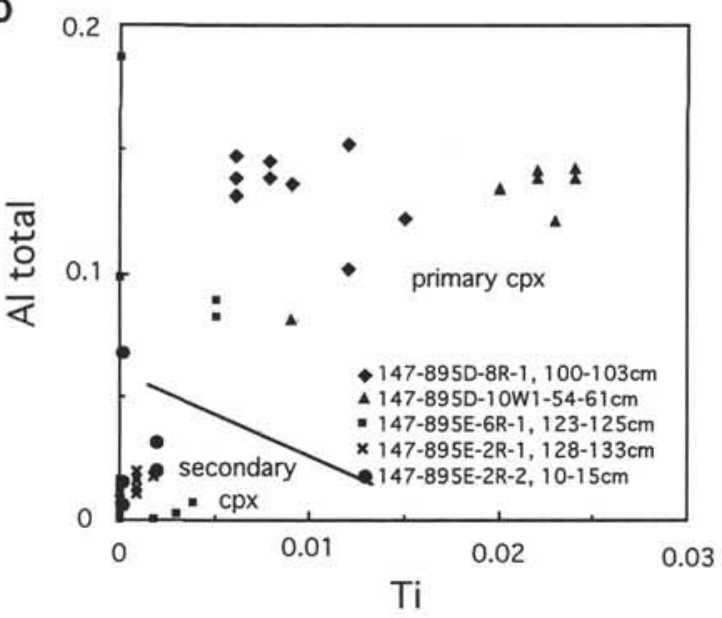

Figure 4. Composition of clinopyroxenes in gabbroic rocks. A. Orientation to area. B. Primary pyroxenes plotted in a Wo:En:Fs ternary diagram, showing the 1 atmosphere solvus (Lindsley, 1983; Lindsley and Andersen, 1983). Filled circles $=$ Sample 147-895D-10W-1, 54-61 cm; filled squares $=$ Sam ple $147-895 \mathrm{E}-6 \mathrm{R}-1,85-87 \mathrm{~cm}$. C. Secondary pyroxenes plotted in the same diagram. Filled circles $=$ Sample $147-895 \mathrm{E}-2 \mathrm{R}-1,128-133 \mathrm{~cm}$; filled squares $=$ Sample $147-895 \mathrm{E}-2 \mathrm{R}-2,10-15 \mathrm{~cm}$; filled triangles $=$ Sample $147-895 \mathrm{E}-$ $6 \mathrm{R}-1,123-125 \mathrm{~cm}$. D. Clinopyroxenes in an $\mathrm{Al}$ vs. Ti diagram. The fields of primary and secondary pyroxenes are clearly distinct.

an early stage. Some of these minerals are strongly enriched in iron, although the chemical environment is magnesium-rich. This iron enrichment suggests that the fluid from which minerals crystallized in veins was very evolved with respect to seawater.

Such a modified fluid phase can only result from reaction with the crust in a rock dominated system. This is consistent with small quantities of fluids reaching the Moho transition zone. These fluids may 
Table 4. Representative analyses of amphiboles.

\begin{tabular}{|c|c|c|c|c|c|c|c|c|c|c|c|c|c|c|c|c|c|c|}
\hline & 1 & 2 & 3 & 4 & 5 & 6 & 7 & 8 & 9 & 10 & 11 & 12 & 13 & 14 & 15 & 16 & 17 & 18 \\
\hline Hole: & 895D & 895D & 895D & 895D & 895D & 895D & $895 \mathrm{E}$ & $895 \mathrm{E}$ & $895 \mathrm{E}$ & $895 \mathrm{E}$ & $895 \mathrm{E}$ & $895 \mathrm{E}$ & $895 \mathrm{E}$ & $895 \mathrm{E}$ & 895E & $895 \mathrm{C}$ & $895 \mathrm{C}$ & $895 \mathrm{C}$ \\
\hline Core, section: & $3 \mathrm{R}-1$ & $7 \mathrm{R}-1$ & $8 \mathrm{R}-2$ & $8 \mathrm{R}-2$ & $8 \mathrm{R}-1$ & OW-1 & IR-1 & IR-1 & IR-1 & IR-3 & $2 \mathrm{R}-1$ & $2 \mathrm{R}-1$ & $2 \mathrm{R}-2$ & $6 \mathrm{R}-1$ & $6 \mathrm{R}-1$ & $4 \mathrm{R}-3$ & $4 \mathrm{R}-3$ & $4 \mathrm{R}-3$ \\
\hline Interval $(\mathrm{cm})$ : & $64-69$ & $56-60$ & $35-38$ & $35-38$ & $100-103$ & $54-61$ & $140-144$ & $140-144$ & $140-144$ & $112-115$ & $128-133$ & $128-133$ & $10-15$ & $85-87$ & $85-87$ & $56-59$ & $56-59$ & $56-59$ \\
\hline Type: & trem & trem & g amph & trem & trem & trem & e amph & $\mathrm{g}$ amph & trem & trem & amph v & trem & $\mathrm{g}$ amph & e amph & trem & Ti-pg & hb & trem \\
\hline $\mathrm{SiO}_{2}$ & 57.71 & 55.55 & 41.98 & 57.54 & 57.42 & 56.64 & 48.28 & 40.46 & 57.46 & 57.08 & 39.86 & 57.98 & 42.28 & 47.95 & 57.57 & 42.47 & 49.17 & 56.56 \\
\hline $\mathrm{TiO}_{2}$ & 0.03 & 0.03 & 0.14 & 0.02 & 0.07 & 0.05 & 0.08 & 0.16 & 0 & 0.02 & 0.09 & 0 & 0.07 & 0.08 & 0.03 & 2.59 & 0.69 & 0.25 \\
\hline $\mathrm{Al}_{2} \mathrm{O}_{3}$ & 0.08 & 1.76 & 14.4 & 0.23 & 0.12 & 0.79 & 11.59 & 15.77 & 0.16 & 1.04 & 16.83 & 0.4 & 13.4 & 11.79 & 0.29 & 12.19 & 6.74 & 1.62 \\
\hline $\mathrm{Cr}_{2} \mathrm{O}_{3}$ & 0.09 & 0.06 & 0 & 0 & 0 & 0 & 0 & 0.04 & 0.02 & 0.09 & 0 & 0.08 & 0 & 0.06 & 0.05 & 0.03 & 0.15 & 0.09 \\
\hline $\mathrm{Fe}_{2} \mathrm{O}_{3}$ & 0 & 0.97 & 3.23 & 0 & 0 & 0.19 & 0 & 3.1 & 0.07 & 0 & 1.79 & 0 & 1.17 & 0 & 0 & 0 & 0 & 0 \\
\hline $\mathrm{FeO}$ & 2.51 & 2.63 & 8.66 & 2.54 & 3.20 & 2.43 & 4.21 & 12.88 & 2.35 & 3.68 & 11.95 & 2.3 & 12.73 & 2.38 & 2.87 & 12.58 & 11.71 & 3.81 \\
\hline $\mathrm{MnO}$ & 0.02 & 0.06 & $\begin{array}{l}0.15 \\
0.15\end{array}$ & 0.08 & 0 & 0.02 & 0.08 & 0.18 & 0.09 & 0.09 & 0.2 & 0.08 & 0.17 & 0.05 & 0.01 & 0.14 & 0.18 & 0.18 \\
\hline $\mathrm{MgO}$ & 23.28 & 22.47 & 12.48 & 22.97 & 22.78 & 23.05 & 18.75 & 9.71 & 23.42 & 21.65 & 10.87 & 23.19 & 11.38 & 19.38 & 22.61 & 12.99 & 14.44 & 21.29 \\
\hline $\mathrm{NiO}$ & 0 & 0.1 & 0.02 & 0.05 & 0.03 & 0.12 & 0.02 & 0.03 & 0.17 & 0.08 & 0.08 & 0.16 & 0.06 & 0.06 & 0.05 & 0.02 & 0 & 0 \\
\hline $\mathrm{CaO}$ & 12.95 & 12.91 & 13.32 & 13.06 & 13.08 & 12.52 & 12.03 & 12.45 & 12.94 & 12.82 & 12.19 & 13.31 & 12.02 & 12.93 & 13.05 & 11.14 & 12.21 & 12.94 \\
\hline $\mathrm{Na}_{2} \mathrm{O}$ & 0.04 & 0.60 & 2.49 & 0.10 & 0.03 & 0.19 & 1.88 & 2.40 & 0.05 & 0.17 & 3.17 & 0.17 & 2.5 & 2.13 & 0.07 & 2.75 & 1.07 & 0.31 \\
\hline $\mathrm{K}_{2} \mathrm{O}$ & 0 & 0.05 & 0.11 & 0.02 & 0 & 0.04 & 0.04 & 0.17 & 0 & 0 & 0.09 & 0 & 0.11 & 0.02 & 0.03 & 0.10 & 0 & 0.03 \\
\hline Total & 96.71 & 97.19 & 96.98 & 96.61 & 96.73 & 96.04 & 96.96 & 97.35 & 96.73 & 96.72 & 97.12 & 97.67 & 95.89 & 96.83 & 96.63 & 97 & & 0.03 \\
\hline $\mathrm{Cl}$ & 0.02 & 0.021 & 0.069 & 0.002 & 0.024 & 0.005 & 0.197 & 0.167 & 0.006 & 0.013 & 0.075 & 0.012 & 0.099 & 0.168 & 0.022 & 0.038 & 0 & 0 \\
\hline $\mathrm{Si}$ & 7.962 & 7.709 & 6.188 & 7.965 & 7.960 & 7.888 & 6.791 & 6.048 & 7.944 & 7.928 & 5.942 & 7.942 & 6.359 & 6.728 & 7.974 & 6.309 & 7.197 & 7.847 \\
\hline $\mathrm{Al}^{\mathrm{iv}}$ & 0.013 & 0.288 & 1.811 & 0.034 & 0.019 & 0.111 & 1.20 & 1.951 & 0.02 & 0.071 & 2.057 & 0.057 & 1.641 & 1.271 & 0.025 & 1.690 & 0.802 & 0.152 \\
\hline $\mathrm{Al}^{\mathrm{V}^{\mathrm{V}}}$ & 0 & 0 & 0.689 & 0.004 & 0 & 0.019 & 0.713 & 0.828 & 0 & 0.099 & 0.899 & 0.007 & 0.735 & 0.679 & 0.023 & 0.434 & 0.360 & 0.113 \\
\hline $\mathrm{Ti}$ & 0.003 & 0.003 & 0.015 & 0.002 & 0.007 & 0.006 & 0.009 & 0.018 & 0 & 0.002 & 0.011 & 0 & 0.008 & 0.008 & 0.003 & 0.288 & 0.076 & 0.026 \\
\hline $\mathrm{Cr}$ & 0.010 & 0.006 & 0 & 0 & 0 & 0 & 0 & 0.005 & 0.002 & 0.010 & 0 & 0.008 & 0 & 0.007 & 0.005 & 0.004 & 0.017 & 0.010 \\
\hline $\mathrm{Fe}^{3+}$ & 0 & 0.101 & 0.358 & 0 & 0 & 0.02 & 0 & 0.349 & 0.008 & 0 & 0.201 & 0 & 0.132 & 0 & 0 & 0 & 0 & 0 \\
\hline $\mathrm{Fe}^{2+}$ & 0.289 & 0.305 & $\begin{array}{l}0.3067 \\
1.067\end{array}$ & 0.294 & 0.37 & 0.283 & 0.495 & $\begin{array}{l}1.61 \\
1.61\end{array}$ & 0.272 & 0.427 & 1.490 & 0.263 & 1.6 & 0.278 & 0.332 & 1.554 & 1.433 & 0.442 \\
\hline $\mathrm{Mn}$ & 0.002 & 0.007 & 0.019 & 0.01 & 0 & 0.003 & 0.010 & 0.023 & 0.010 & 0.010 & 0.025 & 0.009 & 0.021 & 0.006 & 0.001 & 0.017 & 0.023 & 0.021 \\
\hline $\mathrm{Mg}$ & 4.793 & 4.647 & 2.742 & 4.740 & 4.707 & 4.784 & 3.931 & 2.164 & 4.825 & 4.483 & 2.414 & 4.735 & 2.552 & 4.054 & 4.667 & 2.863 & 3.151 & 4.403 \\
\hline $\mathrm{Ni}$ & 0 & 0.011 & 0.002 & 0.006 & 0.004 & 0.013 & 0.00 & 0.003 & 0.01 & 0.009 & 0.01 & 0.018 & 0.008 & 0.007 & 0.006 & 0.003 & 0.001 & 0 \\
\hline $\mathrm{Ca}$ & 1.917 & 1.9 & 2.104 & 1.9 & 1.943 & 1.869 & 1.8 & 1.994 & 1.9 & 1.908 & 1.947 & 1.954 & 1.938 & 1.945 & 1.937 & 1.765 & 1.915 & 1.923 \\
\hline $\mathrm{Na}$ & 0.012 & 0.162 & 0.711 & 0.0 & 0.010 & 0.051 & 0.5 & 0.6 & 0.0 & 0.047 & 0.917 & 0.045 & 0.731 & 0.581 & 0.02 & 0.787 & 0.306 & 0.082 \\
\hline K & 0 & 0.009 & 0.02 & 0.003 & 0 & 0.008 & 0.008 & 0.033 & 0.001 & 0 & 0.017 & 0 & 0.022 & 0.004 & 0.006 & 0.019 & 0.001 & 0.005 \\
\hline Total & 15.018 & 15.174 & 15.732 & 15.028 & 15.026 & 15.06 & 15.498 & 15.73 & 15.044 & 15.002 & 15.935 & 15.043 & 15.754 & 15.576 & 15.007 & 15.741 & 15.289 & 15.032 \\
\hline $\mathrm{Cl}$ & 0.004 & 0.004 & 0.017 & 0 & 0.005 & 0.001 & 0.046 & 0.042 & 0.001 & 0.003 & 0.018 & 0.002 & 0.025 & 0.039 & 0.005 & 0.009 & 0.004 & 0 \\
\hline $\mathrm{XMg}$ & 0.94 & 0.94 & 0.72 & 0.94 & 0.93 & 0.94 & 0.89 & 0.57 & 0.95 & 0.91 & 0.61 & 0.95 & 0.61 & 0.93 & 0.93 & 0.65 & 0.76 & 0.90 \\
\hline
\end{tabular}

Notes: Structural formulac calculated on the basis of 23 oxygens per formula unit. Analysis 1: harzburgite; analyses 2-15: gabbroic rocks; analyses 16-18: diabase dike. trem = tremolite; $\mathrm{g}$ amph = green amphibole; $\mathrm{c}$ amph $=$ early amphibole; Ti-pg = titaniferous pargasite; hb $=$ hornblende. 

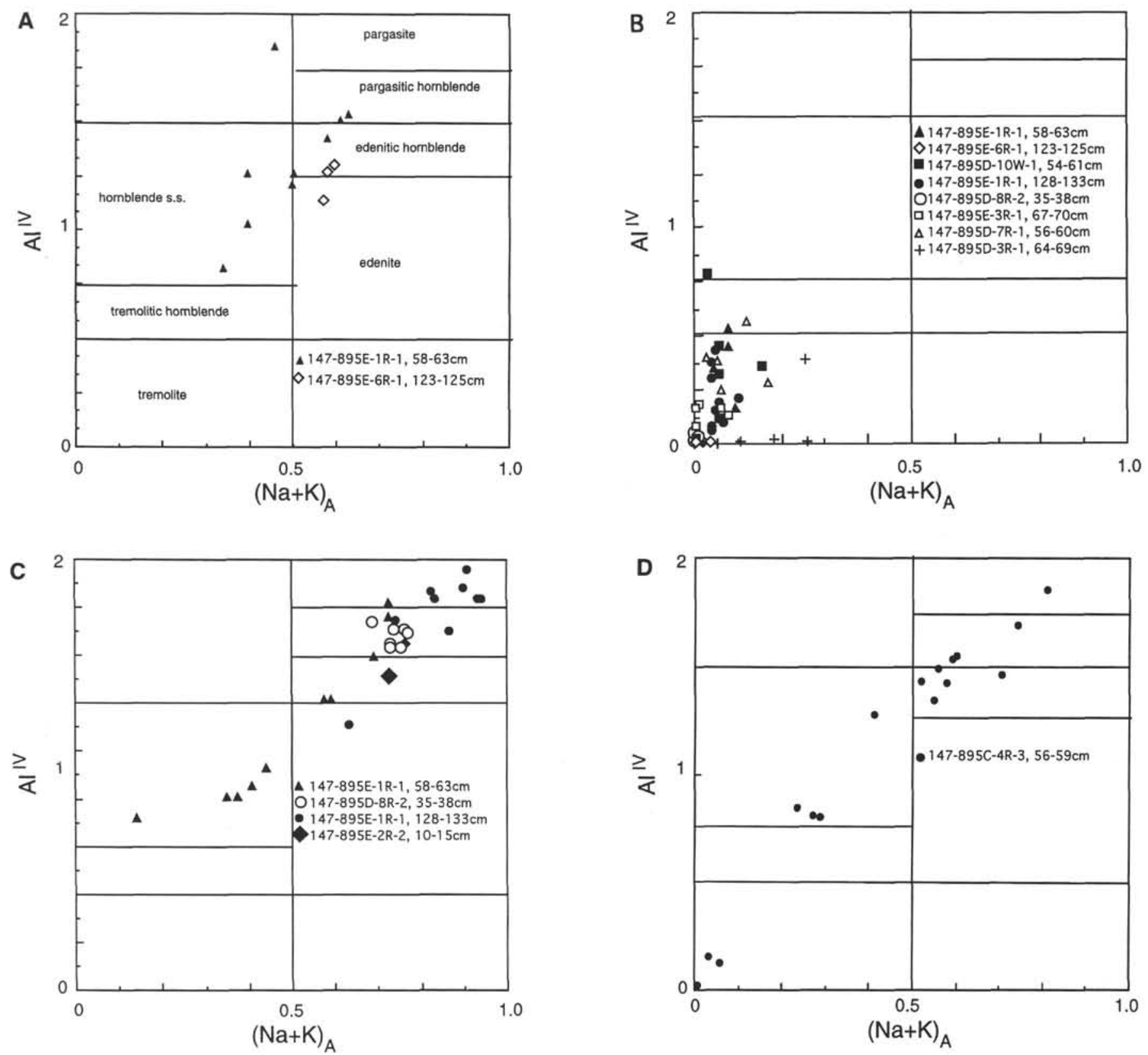

Figure 5. Representation of amphiboles in an $\mathrm{Al}^{\mathrm{IV}} \mathrm{vs} .(\mathrm{Na}+\mathrm{K})_{\mathrm{A}}$ diagram. A. Early, aluminous amphiboles from gabbroic rocks. B. Tremolites from gabbroic rocks and one sample of harzburgite. C. Green, Fe-rich amphiboles from gabbroic rocks. D. Amphiboles from a single sample of diabase.

be the end product of seawater/rock reaction, having penetrated through the whole crust. The association of secondary more-calcic plagioclase with the pyroxene may also result from the composition of the fluid phase (Mével, 1987). This suggests that in this fast spreading environment, only very small quantity of fluid may reach the mantle when it is in its normal position (beneath the magmatic crust).

- The major episode of metamorphism involved a very abundant water-rich fluid phase to produce abundant very hydrous minerals. The mineral-assemblage temperatures are consistent with the parageneses filling the fracture network in Hole $894 \mathrm{G}$. This fracture network, by contrast with veins at Site 895 , shows a very systematic orientation parallel to the strike of the Cocos-Nazca rift and is interpreted to be related to the opening of Hess Deep (MacLeod, Boudier, et al., this volume). Massive penetration of seawater in the mantle may also be related to this event.
-Fluid circulation continued but became less pervasive. Plagioclase was altered to thomsonite, and fluids locally enriched in potassium induced alteration of plagioclase in potassic white mica.

- Late veins were filled with carbonates and possibly clay minerals. This may have happened since the rocks have been exposed on the seafloor.

As opposed to the rocks from the Garrett Fracture Zone, these samples do not record a progressive lowering of temperature but rather massive introduction of seawater at one stage.

\section{Fluxes and Rodingitization}

Rodingites are gabbroic dikes crosscutting peridotites that are enriched in calcium during serpentinization. Rodingites have already been documented on the seafloor (Honnorez and Kirst, 1975; Bideau et al., 1991). Because they contain abundant calc-silicates and seem 


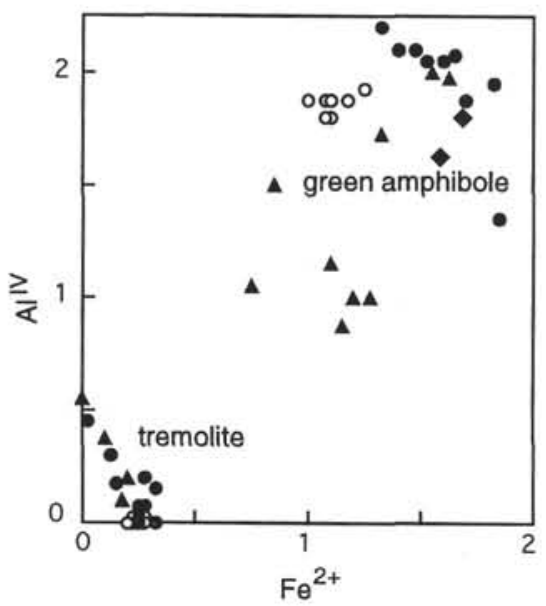

Figure 6. Chemical contrast between the $\mathrm{Al}$-, Fe-poor tremolites and the $\mathrm{Al}$-, Fe-rich ferroan pargasites and hornblendes in gabbroic rocks. Symbols same as in Figure 5C.

devoid of secondary albitic plagioclase, these gabbroic rocks were tentatively considered as rodingites during initial descriptions on board.

The serpentinization of the ultramafics involves a large quantity of hydrous fluids. The gabbros were also extensively hydrated during the same event, compared to gabbroic rocks from the lower crust, as evidenced by the abundance of serpentine, chlorite, and prehnite. It is likely that massive introduction of water induced element fluxes during metamorphism. Documenting calcium enrichment by bulk rock analyses on these samples is problematic because they are very small, with a varying grain size, and because the composition of the protolith is not known for sure- there is no fresh analog. However, using actual mineral compositions, tentative calculations can be made to test whether calcium enrichment is necessary to produce the observed "rodingitic" paragenesis.

We chose a typical example of strongly altered troctolite, Sample 147-895D-7R-1, 56-60 cm, which contains coronas (Fig. 3E). The major episode of metamorphism is characterized by the following corona-forming reaction:

olivine + plagioclase + fluid -----> serpentine + tremolite

+ chlorite + prehnite

As suggested by stable isotope studies (Früh-Green, Plas, and Lécuyer, this volume), the fluid is largely seawater.

We verified that this reaction could be equilibrated without any major input of calcium. We used the actual mineral compositions listed in Table 7 with the exception of the plagioclase. Plagioclase is completely altered in the rock. Since plagioclase composition is rather constant in the troctolites, we used a plagioclase composition from Sample 147-895E-1R-3, 101-104 cm. We considered the seven phases involved in reaction (1), with $\mathrm{H}_{2} \mathrm{O}$ as the fluid phase, and six constituents: $\mathrm{Si}, \mathrm{Al}, \mathrm{Fe}, \mathrm{Mg}, \mathrm{Ca}$, and $\mathrm{H}_{2} \mathrm{O}$. We obtained the following result:

$$
0.36 \mathrm{olv}+0.18 \mathrm{pl}+1 \text { fluid }-\cdots+-0.01 \mathrm{ser}+0.03 \text { trem }
$$

$$
+0.05 \mathrm{chl}+0.02 \mathrm{pr}
$$

In this six-constituent system, the reaction equilibrated without the introduction of calcium. The presence of abundant prehnite in the center of plagioclase pseudomorphs simply reflects a redistribution of chemical elements. To keep the number of constituent equal to the number of phase minus 1 , as required by the phase rule, $\mathrm{Na}_{2} \mathrm{O}$ was not considered in the calculation. However, the initial plagioclase contained some sodium, which enters only tremolite in a small quantity. Most of the sodium is leached during alteration. Therefore, in this case, it seems that the formation of a calc-silicate is more related to an initial very calcic composition (plagioclase is An85-93) and the leaching of sodium rather than an enrichment in calcium. It is only in extreme cases, when chlorite is scarce and most of the plagioclase is replaced by secondary calc-silicates that a calcium enrichment likely occurred.

The formation of the corona texture is related to differential element mobility during metamorphism. Figure 8 displays a sketch of the corona, and the distribution of $\mathrm{Al}, \mathrm{Ca}, \mathrm{Fe}$, and $\mathrm{Mg}$ in the two magmatic phases (olivine and plagioclase) and the four secondary phases (serpentine, tremolite, chlorite, and prehnite) in the reaction. Average compositions are considered. This figure clearly shows that the nature of secondary phases that replace the primary phases is mostly controlled by aluminum, which is known to be the most immobile major element during interaction with seawater under greenschist facies conditions (see Humphris and Thompson, 1978). Aluminum mostly remains on its original site. All the other components are redistributed, in particular the calcium.

A peculiar character of the gabbro suite is the presence of potassic white mica. A number of authors have observed and demonstrated experimentally that at temperatures greater than $150^{\circ} \mathrm{C}$, potassium is leached from rocks (Hajash, 1975; Humphris and Thompson, 1978; Seyfried and Bishoff, 1979). However white mica associated with albite has been described in a few samples from the dike complex at ODP Hole 504B (Laverne, 1987); and in pillow-lavas from the Vema fracture zone (Mével, 1981). White mica is not a low temperature phase: descriptions of oceanic rocks suggest that, at low temperature, potassic phases are zeolites or potassium feldspar. The presence of white mica has to be related to local fluids enriched in potassium, at an undetermined temperature.

The dikes do not seem to have been exposed to the same metamorphic evolution as were the ultramafic and gabbroic rocks. The rocks are generally less recrystallized and display evidence of a progressive lowering of the temperature, starting as high as $700^{\circ} \mathrm{C}$. A possible explanation is that these dikes are late and were emplaced in an already hydrated mantle. An "autometamorphism" due to the heat of the dike combined with abundant fluid would have produced abundant high temperature amphibole. Decrease of the temperature may explain the large range of amphibole compositions (Spear, 1981). It has been suggested that at least part of the dikes crosscutting the lower crust and mantle at Hess Deep are related to the Cocos-Nazca propagator (Francheteau et al., 1990). The dikes displaying this mineral assemblage could correspond to this late emplacement.

\section{Geodynamic Implications}

The present study emphasizes that the major episode of metamorphism required a massive input of seawater in the mantle at temperatures around $300-400^{\circ} \mathrm{C}$, under greenschist facies conditions. Chen and Morgan (1990) have calculated the distribution of isotherms in the oceanic lithosphere as a function of the distance to the ridge axis as well as the spreading rate. The rocks recovered from Site 895 are interpreted to correspond to the transition zone, just beneath the Moho (Boudier et al., this volume). In the EPR at equivalent spreading rate, seismic data suggest a crustal thickness in the order of 5-6 $\mathrm{km}$. Chen and Morgan's (1990) calculations show that at a half spreading rate of $50 \mathrm{~mm} / \mathrm{yr}$, with a thermal conductivity increased by a factor of three to model the hydrothermal cooling, a Moho located at $5-\mathrm{km}$ depth would cross the $500^{\circ}$ isotherm at approximately $30 \mathrm{~km}$ from the ridge axis, and the $400^{\circ} \mathrm{C}$ isotherm even farther. In the com- 
Table 5. Representative analyses of chlorites.

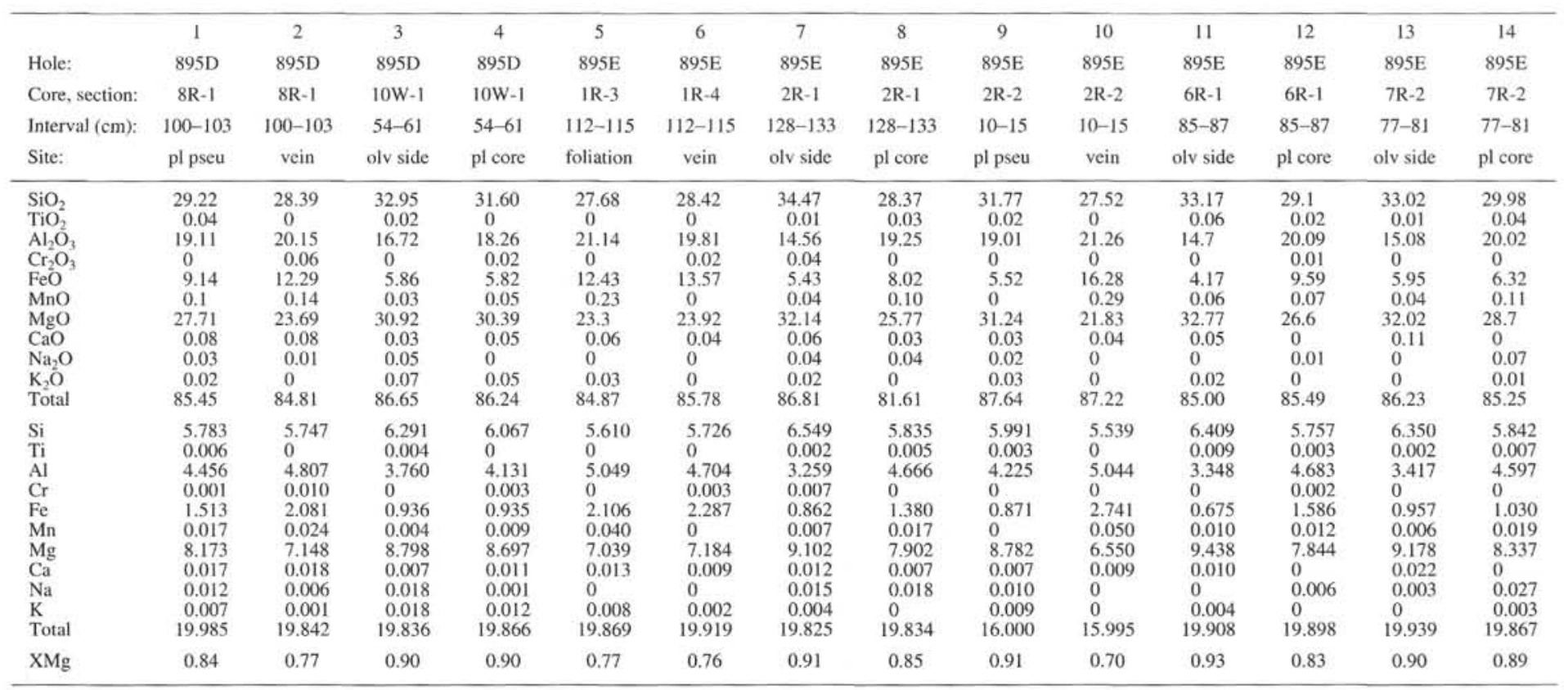

Notes: Structural formulae calculated on the basis of 28 oxygens per formula unit. pl pseu = plagioclase pseudomorph; olv side $=$ olivine side in plagioclase pseudomorph; $\mathrm{pl}$ core $=$ center of plagioclase pseudomorph. 


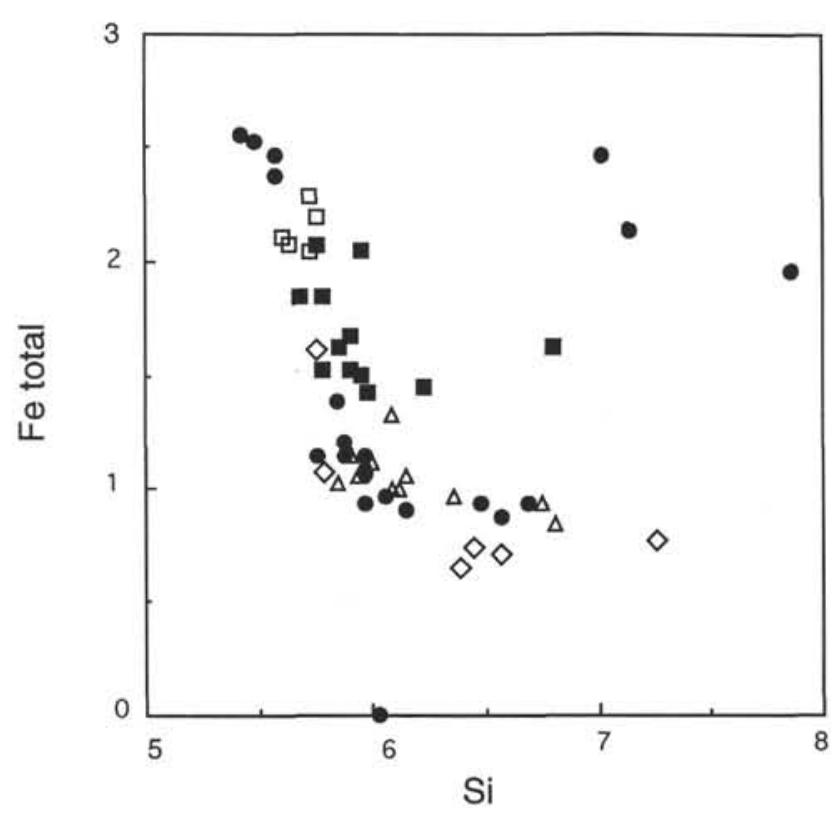

Figure 7. Chemical compositions of chlorites plotted in an Al vs. Fe binary diagram. The chlorites located close to the olivine boundary tend to be enriched in $\mathrm{Si}$ and $\mathrm{Mg}$, as opposed to the chlorites in the center of the plagioclase pseudomorphs. Symbols: open diamonds $=$ Sample 147-895E-6R-1, $123-125 \mathrm{~cm}$; open squares $=$ Sample $147-895 \mathrm{E}-3 \mathrm{R}-1,67-70 \mathrm{~cm}$; solid squares $=$ Sample $147-895 \mathrm{D}-8 \mathrm{R}-1,100-103 \mathrm{~cm}$; solid circles $=$ Sample $147-$ 895E-2R-1, 128-133 cm; open triangles = Sample 147-895D-7R-1, 56-60 $\mathrm{cm}$.

plex tectonic environment at the edge of the Galapagos plate, it is likely that simple thermal models do not apply. However, at $65 \mathrm{~mm} /$ $\mathrm{yr}$, which is the current spreading rate at the adjacent EPR axis, it is possible that the Moho reaches this temperature at the same time as the Cocos-Nazca propagator penetrates the lithosphere. We tentatively propose that massive penetration of seawater in the mantle section is related to rifting initiated by the propagation. By contrast, the early high-temperature metamorphism is very restricted, and may correspond to processes that happened before, at the same time as the high temperature metamorphism in the gabbros (Manning et al., 1993). Such a model has the implication that in the absence of a particular tectonic environment, serpentinization is not widespread in the mantle underlying oceanic crust at a fast-spreading ridge. In this case, the propagation of the Cocos-Nazca rift has allowed seawater penetration; rocks from the Garrett fracture zone (Bideau et al., 1991) show that transform faults may have the same influence.

To explain the uplifting of the intrarift ridge, two models have been proposed (Francheteau et al., 1990). The first one involves diapirism related to serpentinization, the second listric faults due to the opening of the rift. The serpentinite samples drilled at Site 895 display little evidence of deformation. Most serpentinization occurred under static conditions, textures are very well preserved, although it cannot be completely ruled out that more deformed areas were not recovered during drilling. These observations are not consistent with a diapiric uplifting which should have produced sheared serpentinites. A stronger evidence is that serpentine diapirism should result in a mass deficit that lowers the seismic velocities beneath the intra-rift ridge. Seabottom gravity measurements (Hildebrand, pers. comm., 1992) rather suggest a positive anomaly beneath the ridge and the seismic velocities are high beneath the intrarift ridge (Wiggins et al., 1993). This study, together with other observations, therefore suggests that the emplacement of the intrarift ridge is rather related to asymmetric rifting.

\section{ACKNOWLEDGMENTS}

We are thankful to the captain and the crew of Leg 147, who allowed recovery of these rocks in a particularly difficult environment. Discussions with the "Leg 147 metamorphic team," especially Gretchen Früh-Green, as well as with my co-chief Kathryn Gillis were fruitful. The manuscript has greatly benefited from the patient advice of S.C. Komor. This work was supported by a CNRS-INSU "Géosciences Marines" grant. Chrysi Stamoudi is funded by the State Scholarship Foundation of Greece (I.K.Y.).

\section{REFERENCES}

Agrinier, P., Hekinian, R., Bideau, D., and Javoy, M., in press. Stable isotope compositions $\left({ }^{18} \mathrm{O} /{ }^{16} \mathrm{O}, \mathrm{D} / \mathrm{H}\right.$, and $\left.{ }^{13} \mathrm{C} /{ }^{12} \mathrm{C}\right)$ of oceanic crust and upper mantle rocks exposed in the Hess Deep near the Galapagos Triple Junction. Earth Planet. Sci. Lett.

Agrinier, P., Mével, C., and Girardeau, J., 1988. Hydrothermal alteration of the peridotites cored at the ocean/continent boundary of the Iberian Margin: petrologic and stable isotope evidence. In Boillot, G., Winterer, E.L., et al., Proc. ODP, Sci. Results, 103: College Station, TX (Ocean Drilling Program), 225-234.

Bideau, D., Hébert, R., Hekinian, R., and Cannat, M., 1991. Metamorphism of deep-seated rocks from the Garrett ultrafast transform (East Pacific Rise near $13^{\circ} 25^{\prime}$ S). J. Geophys. Res., 96:10079-10099.

Bonatti, E., 1976. Serpentinite protrusions in the oceanic crust. Earth Planet. Sci. Lett., 32:107-113.

Bonatti, E., Lawrence, J.R., and Morandi, N., 1984. Serpentinization of ocean-floor peridotites: temperature dependence on mineralogy and boron content. Earth Planet. Sci. Lett., 70:88-94.

Cannat, M., 1993. Emplacement of mantle rocks in the seafloor at mid-ocean ridges. J. Geophys. Res., 98:4163-4172.

Chen, Y., and Morgan, W.J., 1990. A nonlinear rheology model for MidOceanic Ridge topography. J. Geophys. Res., 95:17583-17604.

Francheteau, J., Armijo, R., Cheminée, J.L., Hekinian, R., Lonsdale, P.F., and Blum, N., 1990. 1 Ma East Pacific Rise oceanic crust and uppermost mantle exposed by rifting in Hess Deep (equatorial Pacific Ocean). Earth Planet. Sci. Lett., 101:281-295.

Francis, T.J.G., 1981. Serpentinization faults and their role on the tectonics of slow-spreading ridges. J. Geophys. Res., 86:11616-11622.

Gillis, K., Mével, C., Allan, J., et al., 1993. Proc. ODP, Init. Repts., 147: College Station, TX (Ocean Drilling Program).

Gillis, K.M., Thompson, G., and Kelley, D.S., 1993. A view of the lower crustal component of hydrothermal systems at the Mid-Atlantic Ridge. J. Geophys. Res., 98:19597-19619.

Girardeau, J., and Francheteau, J., 1993. Plagioclase-wehrlites and peridotites on the East Pacific Rise (Hess Deep) and the Mid-Atlantic Ridge (DSDP Site 339) evidence for magma percolation in the oceanic upper mantle. Earth Planet. Sci. Lett., 115:137-149.

Hajash, A., 1975. Hydrothermal processes along Mid-Ocean Ridges: an experimental investigation. Contrib. Mineral. Petrol., 53:205-226.

Hébert, R., Adamson, A.C., and Komor, S.C., 1990. Metamorphic petrology of ODP Leg 109, Hole 670A serpentinized peridotites: serpentinization processes at a slow spreading ridge environment. In Detrick, R., Honnorez, J., Bryan, W.B., Juteau, T., et al., Proc. ODP, Sci. Results, 106/ 109: College Station, TX (Ocean Drilling Program), 103-115.

Hekinian, R., Bideau, D., Francheteau, J., Cheminée, J.L., Armijo, R., Lonsdale, P., and Blum, N., 1993. Petrology of the East Pacific Rise crust and upper mantle exposed in the Hess Deep (eastern equatorial Pacific). $J$. Geophys. Res., 98:8069-8094.

Honnorez, J., and Kirst, P., 1975. Petrology of rodingites from the Equatorial Mid-Atlantic fracture zones and their geotectonic significance. Contrib. Mineral. Petrol., 49:233-257.

Humphris, S.E., and Thompson, G., 1978. Hydrothermal alteration of oceanic basalts by seawater. Geochim. Cosmochim. Acta, 42:107-125.

Kimball, K.L., Spear, F.S., and Dick, H.J.B., 1985. High temperature alteration of abyssal ultramafics from the Islas Orcadas Fracture zone, South Atlantic. Contrib. Mineral. Petrol., 91:307-320.

Komor, S.C., Elthon, D., and Casey, J.F., 1985. Mineralogic variation in a layered ultramafic cumulate sequence at the North Arm Mountain Massif, Bay of Islands Ophiolite, Newfoundland. J. Geophys. Res., 90:77057736. 
Laverne, C., 1987. Les interactions basalte-fluide en domaine océanique. Minéralogie, pétrologie et géochimie d'un système hydrothermal: le puits 504B, Pacifique oriental [Doctorat d'Etat]. Université d'AixMarseille.

Lindsley, D.H., 1983. Pyroxene thermometry. Am. Mineral., 68:477-493.

Lindsley, D.H., and Andersen, D.J., 1983. A two-pyroxene thermometer. J. Geophys. Res., 88 (Suppl.):A887-A906.

Liou, J.G., Kim, H.S., and Maruyama, S., 1983. Prehnite-epidote equilibria and their petrologic applications. J. Petrol., 24:321-342.

Liou, J.G., Kuniyoshi, S., and Ito, K., 1974. Experimental studies of the phase relations between greenschist and amphibolite in a basaltic system. Am. J. Sci., 274:613-632.

Lonsdale, P., 1988. Structural pattern of the Galapagos microplate and evolution of the Galapagos triple junctions. J. Geophys. Res., 93:1355113574.

Manning, C.E., and the Leg 147 Shipboard Party, 1993. High temperature gabbro metamorphism at Hess Deep and the root of EPR hydrothermal system. Eos, 74:653.

Mével, C., 1981. Occurrence of pumpellyite in hydrothermally altered basalts from the Vema Fracture Zone (Mid-Atlantic Ridge). Contrib. Mineral. Petrol., 76:386-393.

-1987. Evolution of oceanic gabbros from DSDP Leg 82: influence of the fluid phase on metamorphic crystallizations. Earth Planet. Sci. Lett., 83:67-79.

Prichard, H.M., 1979. A petrographic study of the process of serpentinization in ophiolites and the ocean crust. Contrib. Mineral. Petrol, 68:231-241.

Searle, R.C., and Francheteau, J., 1986. Morphology and tectonics of the Galapagos triple junction. Mar. Geophys. Res., 8:95-129.
Seyfried, W.E., Jr., and Bischoff, J.L., 1979. Low temperature basalt-alteration by sea water: an experimental study at $70^{\circ} \mathrm{C}$ and $150^{\circ} \mathrm{C}$. Geochim. Cosmochim. Acta, 43:1937-1948.

Spear, F.S., 1981. An experimental study of hornblende stability and compositional variability in amphibolite. Am. J. Sci., 281:697-734.

Stakes, D., Mével, C., Cannat, M., and Chaput, T., 1991. Metamorphic stratigraphy of Hole 735B. In Von Herzen, R.P., Robinson, P.T., et al., Proc. ODP, Sci. Results, 118: College Station, TX (Ocean Drilling Program), 153-180.

Tartarotti, P., Cannat, M., and Mével, C., 1995. Gabbroic dikelets in serpentinized peridotites from the Mid-Atlantic Ridge at $23^{\circ} 20^{\prime} \mathrm{N}$. In Vissors, R.L.M., and Nicolas, A. (Eds.), Mantle and lower crust exposed in oceanic ridges and in ophiolites: Dordrecht (Kluwer), 35-69.

Vanko, D.A., and Stakes, D.S., 1991. Fluids in oceanic layer 3: evidence from veined rocks, Hole 735B, Southwest Indian Ridge. In Von Herzen, R.P., Robinson, P.T., et al., Proc. ODP, Sci. Results, 118: College Station, TX (Ocean Drilling Program), 181-215.

Wiggins, S.M., Canuteson, E.L., Dorman, L.M., Hildebrand, J.A., Cornuelle, B.D., Sauter, A.W., and Schreiner, A.E., 1993. Crustal structure at the Hess Deep: seismic studies show high velocity in the Intra-Rift ridge and low velocity in the Deep. Eos, 74 (Suppl.):666.

\section{Date of initial receipt: 3 August 1994 \\ Date of acceptance: 27 April 1995 \\ Ms 147SR-017}

Table 6. Representative analysis of prehnite, epidote, thomsonite, and white mica.

\begin{tabular}{|c|c|c|c|c|c|c|c|c|c|c|c|c|}
\hline & 1 & 2 & 3 & 4 & 5 & & 6 & 7 & 8 & 9 & & 10 \\
\hline Hole: & $895 \mathrm{D}$ & $895 \mathrm{D}$ & $895 \mathrm{D}$ & 895D & $895 \mathrm{E}$ & & 895D & $895 \mathrm{E}$ & $895 \mathrm{E}$ & $895 \mathrm{E}$ & & $895 \mathrm{E}$ \\
\hline Core, section: & $7 \mathrm{R}-1$ & $8 R-1$ & $10 \mathrm{~W}-1$ & $10 \mathrm{~W}-1$ & $2 \mathrm{R}-1$ & & $8 \mathrm{R}-1$ & $2 \mathrm{R}-1$ & IR-1 & $2 \mathrm{R}-2$ & & $2 \mathrm{R}-1$ \\
\hline Interval $(\mathrm{cm})$ : & $56-60$ & $100-103$ & $54-61$ & $54-61$ & $128-133$ & & $100-103$ & $128-133$ & $140-144$ & $10-15$ & & $128-133$ \\
\hline Mineral: & $\mathrm{pr}$ & $\mathrm{pr}$ & $\mathrm{pr}$ & ep & $\mathrm{pr}$ & & thom & thom & thom & thom & & mica \\
\hline $\mathrm{SiO}_{2}$ & 42.34 & 41.14 & 43.02 & 42.47 & 42.49 & $\mathrm{SiO}_{2}$ & 37.99 & 36.6 & 38.68 & 37.5 & $\mathrm{SiO}_{2}$ & 45.98 \\
\hline $\mathrm{TiO}_{2}$ & 0.00 & 0 & 0.01 & 0 & 0 & $\mathrm{Al}_{2} \mathrm{O}_{3}$ & 30.05 & 30.56 & 29.94 & 30.49 & $\mathrm{TiO}_{2}$ & 0 \\
\hline $\mathrm{Al}_{2} \mathrm{O}_{3}$ & 23.79 & 26.43 & 24.6 & 32.13 & 24.12 & $\mathrm{FeO}$ & 0 & 0.07 & 0.14 & 0 & $\mathrm{Al}_{2} \mathrm{O}_{3}$ & 34.73 \\
\hline $\mathrm{Fe}_{2} \mathrm{O}_{3}$ & 0.09 & 0.01 & 0.04 & 0 & 0.02 & $\mathrm{MgO}$ & 0.01 & 0.02 & 0.06 & 0 & $\mathrm{FeO}$ & 0.1 \\
\hline $\mathrm{MnO}$ & 0 & 0 & 0 & 0.08 & 0 & $\mathrm{CaO}$ & 12.69 & 13.24 & 15.7 & 12.72 & $\mathrm{MnO}$ & 0.08 \\
\hline $\mathrm{MgO}$ & 0.23 & 0.03 & 0.03 & 0.01 & 0.06 & $\mathrm{Na}_{2} \mathrm{O}$ & 3.73 & 3.64 & 1.44 & 3.59 & $\mathrm{MgO}$ & 1.76 \\
\hline $\mathrm{CaO}$ & 26.17 & 23.61 & 26.02 & 21.14 & 25.63 & $\mathrm{~K}_{2} \mathrm{O}$ & 0 & 0.01 & 0.04 & 0.00 & $\mathrm{CaO}$ & 0.21 \\
\hline $\mathrm{Na}_{2} \mathrm{O}$ & 0.02 & 0.55 & 0.08 & 0.47 & 0.19 & Total & 84.47 & 84.15 & 86.05 & 84.78 & $\mathrm{Na}_{2} \mathrm{O}$ & 0.48 \\
\hline \multirow[t]{2}{*}{ Total } & 92.64 & 91.77 & 93.8 & 96.3 & 92.51 & & & & & & $\mathrm{~K}_{2} \mathrm{O}$ & 9.28 \\
\hline & & & & & & $\mathrm{Si}$ & 5.712 & 5.047 & 5.198 & 5.118 & Total & 92.62 \\
\hline $\mathrm{Si}$ & 6.000 & 5.839 & 6.004 & 3.217 & 6.014 & $\mathrm{Al}$ & 5.325 & 4.966 & 4.742 & 4.904 & & \\
\hline $\mathrm{Ti}$ & 0 & 0 & 0.001 & 0 & 0 & $\mathrm{Fe}$ & 0.002 & 0.008 & 0.014 & 0 & $\mathrm{Si}$ & 6.213 \\
\hline $\mathrm{Al}$ & 3.974 & 4.422 & 4.046 & 2.868 & 4.024 & $\mathrm{Mg}$ & $\quad 0.002$ & 0.004 & 0.012 & 0 & $\mathrm{Ti}$ & 0 \\
\hline $\mathrm{Fe}^{3+}$ & 0.010 & 0.001 & 0.02 & 0 & 0.002 & $\mathrm{Ca}$ & 2.044 & 1.955 & 2.261 & 1.921 & $\mathrm{Al}$ & 5.531 \\
\hline Mn & 0 & 0 & 0 & 0.005 & 0 & $\mathrm{Na}$ & 1.087 & 0.974 & 0.376 & 0.951 & $\mathrm{Fe}$ & 0.012 \\
\hline $\mathrm{Mg}$ & 0.049 & 0.007 & 0.007 & 0.002 & 0.013 & $\mathrm{~K}$ & 0 & 0.001 & 0.007 & 0.000 & $\mathrm{Mn}$ & 0.009 \\
\hline $\mathrm{Ca}$ & 3.974 & 3.591 & 3.89 & 1.717 & 3.887 & Total & 14.172 & 12.958 & 12.614 & 12.902 & $\mathrm{Mg}$ & 0.354 \\
\hline $\mathrm{Na}$ & 0.005 & 0.152 & 0.023 & 0.070 & 0.054 & & & & & & $\mathrm{Ca}$ & 0.03 \\
\hline \multirow[t]{3}{*}{ Total } & 14,011 & 14.012 & 13.991 & 7.882 & 13.998 & & & & & & $\mathrm{Na}$ & 0.127 \\
\hline & & & & & & & & & & & $\mathrm{K}$ & 1.600 \\
\hline & & & & & & & & & & & Total & 13.879 \\
\hline
\end{tabular}

Notes: Structural formulae calculated on the basis of 22 oxygens and total iron as $\mathrm{Fe}_{2} \mathrm{O}_{3}$ for prehnite, 12.5 oxygens and total iron a s $\mathrm{Fe} \mathrm{O}_{3}$ for epidote, 20 oxygens and total iron as $\mathrm{FeO}$ for thomsonite, 22 oxygens and total iron as $\mathrm{FeO}$ for white mica. $\mathrm{pr}=$ prehnite; $\mathrm{ep}=$ epidote; thom = thomsonite; mica = white mica. 
Table 7. Mineral compositions used to balance reaction 1.

\begin{tabular}{lcccccc}
\hline & olv & pl & ser & trem & chl & pr \\
\hline $\mathrm{SiO}_{2}$ & 40.31 & 46.11 & 40.71 & 55.55 & 30.25 & 42.34 \\
$\mathrm{Al}_{2} \mathrm{O}_{3}$ & 0 & 33.9 & 2.34 & 1.76 & 18.69 & 23.79 \\
$\mathrm{FeO}$ & 10.17 & 0 & 4.63 & 2.63 & 6.92 & 0 \\
$\mathrm{MnO}$ & 48.48 & 0 & 37.53 & 22.47 & 29.26 & 0 \\
$\mathrm{CaO}$ & 0 & 17.73 & 0 & 12.91 & 0 & 26.17 \\
$\mathrm{Na}_{2} \mathrm{O}$ & 0 & 1.53 & 0 & 0.6 & 0 & 0 \\
\hline
\end{tabular}

Notes: olv = olivine; $\mathrm{pl}=$ plagioclase, $\mathrm{ser}=$ serpentine $;$ trem = tremolite chl = chlorite; $\mathrm{pr}=$ prehnite. All compositions are from Sample 147-895D-7R-1, 56-60 cm, except the plagioclase, which is from Sample 147-895E-IR-3, 112-115 cm.

FRESH
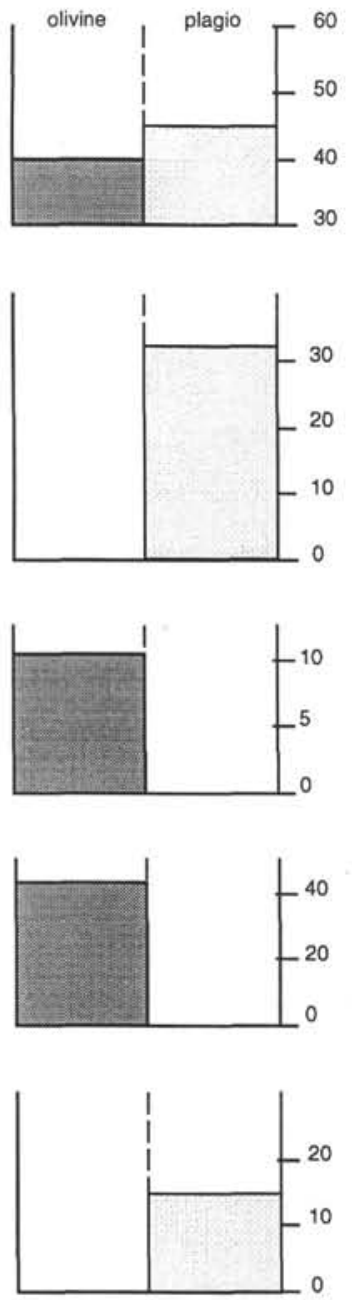

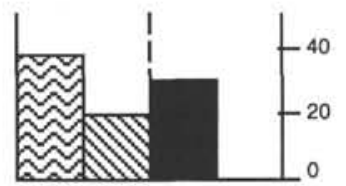

$\mathrm{MgO}$

$\mathrm{SiO}_{2}$
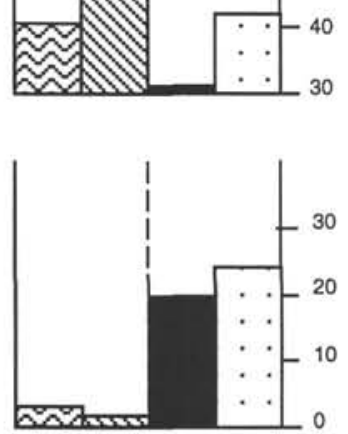

$\mathrm{Al}_{2} \mathrm{O}_{3}$

$\mathrm{FeO}$
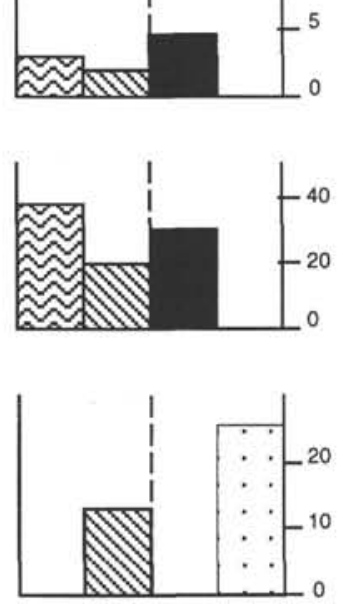

$\mathrm{CaO}$

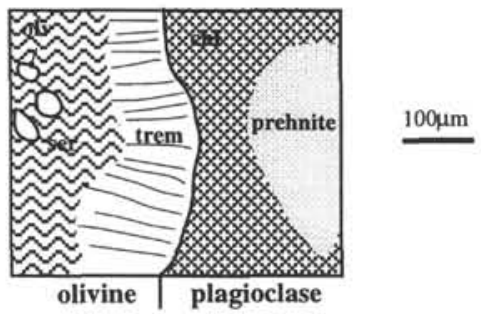

Figure 8. Representation of the redistribution of chemical elements during the corona formation (abbreviations as in Table 1). The analyses used are listed in Table 7. 